\title{
Enhanced Multi-Objective Particle Swarm Optimisation for Estimating Hand Postures
}

\author{
Shahrzad Saremi, Seyedali Mirjalili*, Andrew Lewis, Alan Wee-Chung Liew, Jin Song Dong \\ Institute for Integrated and Intelligent Systems, Griffith University, Brisbane, 4111 QLD, Australia \\ ${ }^{*}$ seyedali.mirjalili@griffithuni.edu.au
}

\begin{abstract}
Multi-objective problems with conflicting objectives cannot be effectively solved by aggregation-based methods. The answer to such problems is a Pareto optimal solution set. Due to the difficulty of solving multi-objective problems using multi-objective algorithms and the lack of enough expertise, researchers in different fields tend to aggregative objectives and use single-objective algorithms. This work is a seminal attempt to propose the use of multi-objective algorithms in the field of hand posture estimation. Hand posture estimation is a key step in hand gesture recognition, which is a part of an overall attempt to make human-computer interaction more like human face-to-face communication. Hand posture estimation is first formulated as a bi-objective problem. A modified version of Multi-Objective Particle Swarm Optimisation (MOPSO) is then proposed to approximate the Pareto optimal font of 50 different postures. The main motivation of integrating a new operator (called Evolutionary Population Dynamics - EPD) in MOPSO is due to the nature of hand posture estimation problems in which parameters should not be tuned in a same manner since they show varied impacts on the objectives. EPD allows randomising different parameters in a solution and provides different exploratory behaviours for the parameters of an optimisation algorithm rather than each individual solution. The MOPSO algorithm is equipped with a mechanism to randomly re-initialise poor particles around the optimal solutions in the archive. The improved MOPSO is tested on ZDT and CEC2009 test functions and compared with the standard MOPSO, NSGA-II, and MOEA/D. The results show that the proposed MOPSO (MOPSO+EPD) significantly outperforms MOPSO on the majority of test functions in terms of both convergence and coverage. MOPSO+EPD also approximates well-distributed Pareto optimal fronts for most of the postures considered in this work. The post analysis of the results is conducted to understand the relationship between the parameters and objectives of this problem (design principals) for the first time in the literature as well.
\end{abstract}

Keywords: Multi-objective problems, Multi-objective optimisation, Multi-Objective Particle Swarm Optimisation, MOPSO, Hand Posture Estimation

\section{Introduction}

Evolutionary Algorithms (EA) [1] have been popular technique to find optimal solutions for optimisation problems. Such algorithms are reliable alternatives compared to conventional gradient-based algorithm mostly due to a better global search. This originates from population-based nature of EAs. In population-based algorithms, a population of candidate solutions is improved by the algorithm. This results in a better exploratory behaviour of an algorithm compared to algorithms with one candidate solution. The consideration of a problem as a black box is another advantage of EAs. The internal equations used in the problem is not essential for EAs to operator, making them suit- able for real-world problems with unknown search landscape. By contrast, conventional gradient-based algorithms require calculating the derivation of search landscape.

Despite the advantages of EAs, optimisation of realworld problems using them involves several difficulties: multi-modality [2], local optima [3], constraints [4], varying parameters type [5], multiple objectives [5], and uncertainties [6]. Local optima are sub-optimal and should be avoided by an algorithm. Note that all global solutions are locally optimum in their neighbourhoods, but sub-optimal solutions compared to the global optimum is not desirable when solving a problem. Real problems have a large number of local optima which 
makes finding the global optimum very challenging for optimisation problems. The existence of multiple global solutions (multi-modality) is another challenge. Finding and differentiating multiple optimal solutions requires special mechanism for an algorithm. Constraints are the restrictions applied to the problem and should not be violated by a solution. An algorithm should be able to avoid infeasible solution that violate constraints. The parameters of optimisation problem might be of different types and treated differently by an algorithm. Multiple objective is another difficulty since such problems have a set of optimal solutions. An optimisation algorithm needs to find an accurate estimation of true Pareto optimal front with high distribution across all objectives. Finally, uncertainties occur when operating in a real environment and provide undesirable inputs (noises) in the system. Finding an optimal solution tolerant to these changes is challenging.

This work tackles the problem of hand posture estimation [7] which requires handling five difficulties [8] simultaneously as follows:

- Local optima: due to the large number of controlling parameters in 3D hand models, there search space is massive. Due to the similarity of poses, there is a significant number of local optima as well.

- Constraints: 3D hand models requires a lot of constrains and limitations to simulate the kinematic movements in the hand. Such constraints limit the search space and make a lot of solutions of an optimisation algorithm infeasible.

- Parameters with different importance: The translation, rotation, and skeleton movements are defined using different parameters. The impact of these sets of parameters on the objective function is different that should be considered.

- Non-derivative objective function: the objective functions of the problem of hand posture estimation are made of a large number of mathematical models, which makes calculating the derivation of them very difficult or impossible. Therefore, gradient-based optimisation techniques cannot be used unless the objective function be simplified.

- Multiple objectives: The hand posture estimation process involves minimising the cost of calculating objective function and maximizing the accuracy of the model. These two objectives are in conflict that should be addressed when estimation hand postures.
Due to these feature of the hand posture estimation problem, the conventional optimisation techniques including the gradient-based approaches are not effective. This is because most of such methods require derivation of the problem and suffer from local optimal stagnation. Addressing conflicting objectives also requires a multiobjective algorithm. These are the motivation of using a stochastic multi-objective algorithm (MOPSO) in this work to find the optimal solutions for this problem.

Hand posture estimation is an important core step when recognizing hand gestures. This refers to the process of finding the accurate pose using a hand model in the computer that represents the real hand model as accurately as possible. It is a key phase since an inaccurate model negatively impacts other core steps of hand gesture recognition: feature extraction and classification.

In this work, the problem of hand posture estimation is formulated considering two objectives for the first time in the literature: minimising the cost of objective function (minimising the number of points in the point cloud required to calculate the accuracy of a 3D hand model) and maximising accuracy of the 3D model. An improved Multi-Objective Particle Swarm Optimisation (MOPSO) [9], equipped with two operators to handle parameters of different type and constraints, is proposed to estimate the Pareto optimal front for the hand posture estimation problem. The rest of the paper is organised as follows:

Section 2 reviews the literature of hand posture estimation and evolutionary multi-objective algorithms. The MOPSO+EPD is proposed in Section 3. Section 4 is dedicated to the presentation and analysis of the proposed algorithm's results on a set of optimisation test functions. Section 5 employs the proposed algorithm to find the Pareto optimal front for 50 postures. Finally, Section 6 concludes the work and discuss future works.

\section{Literature review}

There are four core steps for recognizing hand gestures in computer [10]: image acquisition, hand modelling (estimation), feature extraction, and classification. In the first core step, data is obtained from an input device. In vision-based gesture recognition, the input device is RGB or depth camera. To improve the accuracy in this phase, hardware and technology should be improved. However, the rest of core steps can be improved with effective computer programs and algorithms. After obtaining the image of hand, the second core step is to find an accurate hand model to match with the hand in the image (see Fig. 1). Depending on the hand model, a set of important features are selected 


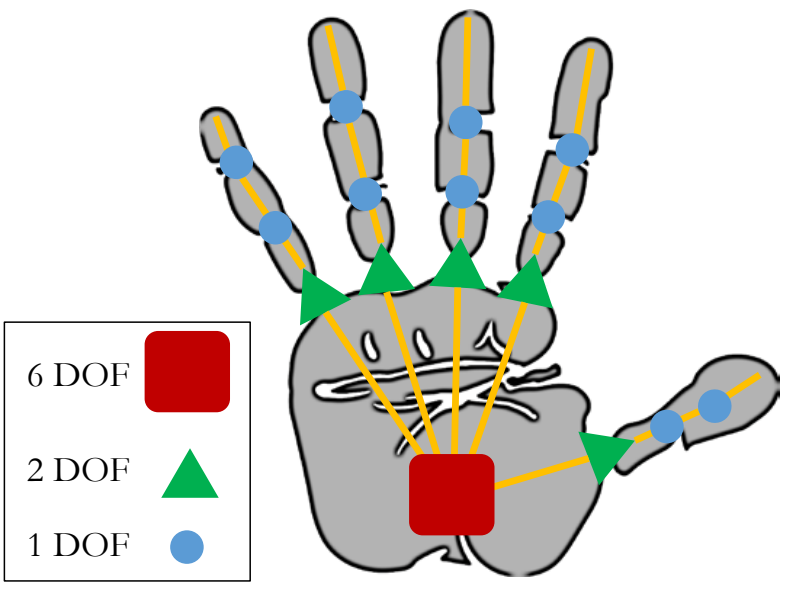

Figure 1: An example of hand kinematic model with 26 DOF

from the model to reduce the dimension of problem and facilitate the process of classification. The last key process is to recognise the hand posture/gesture using the features. The main purpose of classification techniques is to assign each combination of features to a meaningful gesture. This section provides the literature review of hand posture estimation, which is one of the most important activities in the second core step. Finding an accurate hand model is a stepping stone to design an effective hand gesture detection system.

\subsection{Hand posture estimation}

Hand posture estimation techniques may be divided into three main categories in the literature: generative, discriminative, and hybrid.

\subsubsection{Generative techniques}

In such methods, sometimes called model-matching, a hand model is generated and matched with the real hand posture $[11,12]$. The model can be made of a mesh or simple components (sphere, cylinder, etc.). Each hand posture can be determined by a set of parameters for the hand model. An optimisation algorithm is required to find the optimal values for the hand model. The advantage of generative methods is flexibility to a variety of hand poses, ability to consider previous hand postures, and ability to model both discrete and continuations poses. All the benefits come from the modelgenerative nature of such techniques. As a drawback, however, finding an optimal hand model is computationally expensive, In addition, generative methods are sensitive to initial poses and different hand sizes.

Generative methods were systematically proposed by Oikonomidis et al. [13], in which they attempted to find a solution to the difficulty of recovering and tracking 3D position, orientation and all different possibilities of hand pose, when using Kinect sensor and optimisation techniques. They tackled these difficulties as an optimisation problem. They tried to solve this problem by using Particle Swarm Optimisation (PSO) [14]. Despite the merits of this work, hand pose estimation is not smooth and the optimisation suffers from local optima stagnation. They would have to use parallel processing or GPU to achieve 30 FPS (real-time) when modelling hand [13]. Achieving a high FPS when modelling hands is important since it is one of the most computationally expensive steps in the process of hand gesture recognition. The sensitivity to the initial pose especially to start the estimation process was another issue. Consequently, the system was not able to accurately recover the hand model in case of wrong hand tracking.

To tackle the disadvantages of Oikonomidis et al. work, Qian et al. [15] proposed a re-initialiser that assists PSO in starting with a promising initial posture. In this method, fingertips are first found and used to assist PSO. This means that they hybridized model-based and template-based techniques to find a promising initial point. Generally speaking, the main drawbacks of generative techniques is the sensitivity to hand size and the simplicity of model. In addition, in a considerable number of cases, the initial pose is not highly accurate, and the user has to wear a black or blue wrist band for hand segmentation [16].

\subsubsection{Discriminative methods}

Discriminative or template-matching methods do not manipulate a hand model [17]. Such methods work with the image and a database of hand poses. The first step is to create a database of postures. For any given hand images, segmentation and feature extraction should be done to find the closest posture in the database [18]. The use of image and dataset makes discriminative methods computationally less expensive than generative methods. Also, the initial pose is not longer important. However, they are restricted to the hand poses in the dataset and consequently less flexible in predicting unseen hand poses. As such, discrete poses can be only estimated [19].

In 2016, an appearance-based method was proposed using point cloud method [20]. The main objective was to minimise the distance between point cloud obtained by a 3D model and the point cloud generated form the depth image. The main problem was the consideration of only six gestures.

In the literature, there are discriminative methods that use very flexible hand models. For instance, Ballan et 
al. [21] proposed a technique using salient points fitting a 3D model and the hand posture obtained from a camera. The concentrated on one and two hands and got accurate results. However, the method (especially the objective function) was computationally very expensive. The use of multiple cameras, which make this method less practical, was another drawback.

\subsubsection{Hybrid methods}

Due to the drawbacks of both generative and discriminative methods, hybrid methods have been popular. As the name implies, hybrid methods hybridize two techniques. A discriminative algorithm is first used to find the closest posture in the database. A generative algorithm is then used to improve the accuracy of hand model [22].

In 2015, Sharp et al. [23] proposed a hybrid method using fast learned re-initialisation and model fitting technique based on PSO. Also, they used a new objective function to be calculated in GPU. They designed a very fast, accurate, and robust system that shows very competitive results compared to the state-of-theart. However, the system only tracks a single hand and sensitive to occlusion. They heavily relied on GPU, which make them less practical for regular computers and especially mobile devices.

In 2015, Krejov et al. [24] utilised a hybrid method as well. They used A Randomised Decision Forests (RDF) to find an initial pose and then Rigid Body Dynamics to find the optimal shape for hand. The main drawback of this method is the local optima stagnation due to the use of only one method. Also, the segmentation might be computationally expensive. They did not use multiple models in each iteration and there was only one model that evolved to fit the hand projection.

In 2016, Taylor et al. [25] tried to reduce the computational cost of hand posture estimation when using a complicated hand mesh. They used a model with smooth surface and find its optimal shape with Levenberg optimisation technique. A new initialization technique and modification of the objective function (energy function) were other contributions. Despite their contributions, the system was sensitive to heavy occlusion and not able to work with images with complicated backgrounds. The system itself was also super complicated and difficult to replicate mainly due to the use of a nonstandard customised hand model and lack of implementation details.

In 2015 by Sridhar et al. [26] used a decision frost classifier as a discriminative method and local gradientbased optimisation with robust particle-based technique as the generative method. It is interesting that they used two solutions and 10 iterations only. Despite the good frame rate, however, the method did not perform well for fast movement and high articulation. This is due to the used of a gradient-based method. Although they tried to improve it by introducing randomness, they failed to resolve local optima stagnation completely. This can be confirmed since they method failed when there is a large global rotation.

An outstanding recent hybrid work was done by Tkach et al. in 2016 [27]. They proposed a spheremesh model for articulated tracking. The tracking is done with generative method and the assistance of a gradient-based optimisation algorithm (lsqnonlin Matlab routine). The authors also proposed two metrics, which were algorithm independent, for quantifying the performance of algorithms. The re-initialization was simple. The calibration [28] of the hand model parameters was also very effective for different hand sizes. Also, the gradient-based nature of the optimiser makes this method prone to local optima stagnation.

Despite the recent advances in the three main categories, hand posture estimation is still an open research question. There is a large number of challenges that should be tackled to completely answer this research question including the large number of variables to optimise, the expensive objective function, and the large number of constraints. This paper concentrates on generative methods since they are the most flexible techniques in the literature. It is tried to design a generative method considering multiple objectives for the first time. Another gap that has been investigated little in the literature is the use of priority-based optimisation algorithms to tune the parameters of hand models in different rates.

\subsection{Evolutionary multi-objective optimisation}

In contrast to single-objective optimisation, multiobjective optimisation considers more than one objective. Due to the existence of multiple conflicting objectives, there is no longer one solution as the best solution for a problem with multiple objectives. The existence of multiple objectives requires new orators for an optimisation algorithm to compare solutions in the search space. Without loss of generality, multi-objective optimisation is formulated as a minimisation problem as follows [29]:

$$
\begin{gathered}
\text { Minimise : } \quad F(\vec{x})=\left\{f_{1}(\vec{x}), f_{2}(\vec{x}), \ldots, f_{o}(\vec{x})\right\} \\
\text { Subject to }: \quad g_{i}(\vec{x}) \geq 0, i=1,2, \ldots, m \\
h_{i}(\vec{x})=0, i=1,2, \ldots, p
\end{gathered}
$$




$$
l b_{i} \leq x_{i} \leq u b_{i}, i=1,2, \ldots, n
$$

where $o$ is the number of objective functions, $m$ is the number of inequality constraints, $p$ is the number of equality constraints, $g$ shows the inequality constraints, $h$ indicates the equality constraints, and $\left[l b_{i}, u b_{i}\right]$ are the boundaries of the $i$-th variable.

In order to compare two solutions in a multi-objective problem, relational operators are not effective. Pareto optimal dominance is the operator to use for such problems [30, 31]. Pareto optimality is defined as follows [32]:

Definition 2.1. (Pareto Optimality): A solution $\vec{x} \in X$ is called Pareto-optimal iff:

$$
\{\nexists \vec{y} \in X \mid \vec{y}<\vec{x}\}
$$

In a minimisation problem, two solutions can be compared using Pareto dominance as follows:

Definition 2.2. (Pareto Dominance): Suppose that there are two vectors such as: $\vec{x}=\left(x_{1}, x_{2}, \ldots, x_{k}\right)$ and $\vec{y}=\left(y_{1}, y_{2}, \ldots, y_{k}\right)$.

Vector $\vec{x}$ dominates vector $\vec{y}$ (denote as $\vec{x}<\vec{y}$ ) iff:

$$
\begin{gathered}
\forall i \in(1,2, \ldots, o) \\
{\left[f_{i}(\vec{x}) \leq f_{i}(\vec{y})\right] \wedge\left[\exists i \in 1,2, \ldots, o: f_{i}(\vec{x})<f_{i}(\vec{y})\right]}
\end{gathered}
$$

The above definition can be extended to maximisation problems with changing $<$ and $\leq$ to $>$ and $\geq$. This definition shows that in a multi-objective search space, a solution is better than another if and only if it does not show worse value in any of the objectives. In other words, a solution should show equal values in all objectives and better value in at least one of the objectives. Fig. 2 shows the concept of Pareto dominance in a bi-objective search space. This figure illustrates the parameter space and objective space. Pareto optimal dominance is calculated based on the objective space. Inspecting this figure, the blue diamonds both dominate the red triangle because they both show less value along both $f_{1}$ and $f_{2}$.

Note that the diamonds do not dominate each other and they are Pareto optimal solutions for this problem. In other words, they are non-dominated with respect to each other. This is because they are both dominated in one of the objectives. If we consider one objective only, one of them will be chosen as the best solution. However, they are both good solutions (Pareto optimal) for the multi-objective problem.

Every multi-objective problem has a set of nondominated solutions that represents the best trade-offs between the objectives. This set is the answer for a multi-objective problem and defined as follows:

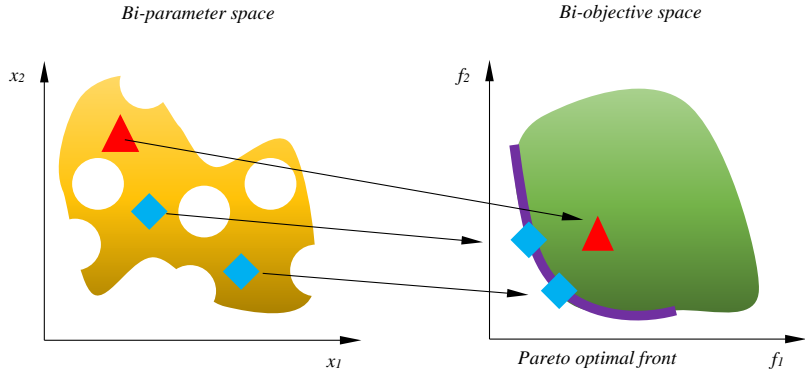

Figure 2: Pareto optimal solution, Pareto optimal front, nondominated solution, and non-dominated solution

Definition 2.3. (Pareto optimal set): The set of all Pareto-optimal solutions:

$$
P S:=\{\vec{x}, \vec{y} \in X \mid \nexists \vec{y}<\vec{x}\}
$$

The Pareto optimal set is found in the parameter space. The projection of the Pareto optimal solutions in the objective space is called Pareto optimal front and defined as follows:

Definition 2.4. (Pareto optimal front): A set containing the value of objective functions for Pareto solutions set:

$$
\begin{gathered}
\forall i \in(1,2, \ldots, o) \\
P F:=\left\{f_{i}(\vec{x}) \mid \vec{x} \in P S\right\}
\end{gathered}
$$

The Pareto optimal front is shown in the right subplot of Fig. 2. It can be seen that there is no better solutions than the Pareto optimal front. Every multiobjective problem has a true Pareto optimal front. The parameters have direct influence on the objective space. The reason is due to the existence of one set of objectives for every unique combination value for the parameters when using the objective function. In other words, the objective function maps the combination of parameters in the parameter space into the objective space.

In the literature of evolutionary multi-objective optimisation, there are three main categories of techniques to approximate Pareto optimal solutions: aggregative methods, posteriori methods, and interactive methods. In the first category, the objectives are aggregated using a set of weights that defines the importance of each objective from a decision makers' point of view. The following equation shows a the general for of aggregation method:

Minimise: $\quad F(\vec{x})=w_{1} f_{1}(\vec{x})+w_{2} f_{2}(\vec{x})+\ldots+w_{o} f_{o}(\vec{x})$

The main advantage of this technique is the use of single-objective algorithms. However, the algorithm 
needs to be run multiple times and weights should be changed to find the Pareto optimal set. Another drawback is that some type of Pareto optimal fronts (concave) cannot be found by such techniques.

Posteriori methods alleviate the drawbacks of aggregation methods by maintaining the multi-objective formulation and estimating Pareto optimal solution set in a single run. This means that the decision making is done after optimisation. However, an algorithm should be equipped with suitable operators and mechanisms to maintain and improve estimated Pareto optimal solutions during the optimisation process. Such techniques need to find a uniformly distributed solutions across all objectives to provide decision makes with a diverse range of solutions.

Interactive methods can be used in conjunction with aggregative or posteriori approaches. In such techniques, optimisation process is conducted with direct involvements of decision makers. An optimisation algorithm in this category pauses the optimisation process occasionally (or periodically) to consider and apply decision makers' inputs. Therefore, decision makers have the opportunity to direct an algorithm towards desired Pareto optimal solutions.

The focus of this work is on the second type of multiobjective optimisation since decision making is out of the scope of this work. We attempt to estimate the Pareto optimal front for several hand postures and leave the decision making to a future work. In the next subsection, the details of the MOPSO algorithm are given since it is the main posteriori optimisation algorithm in this work.

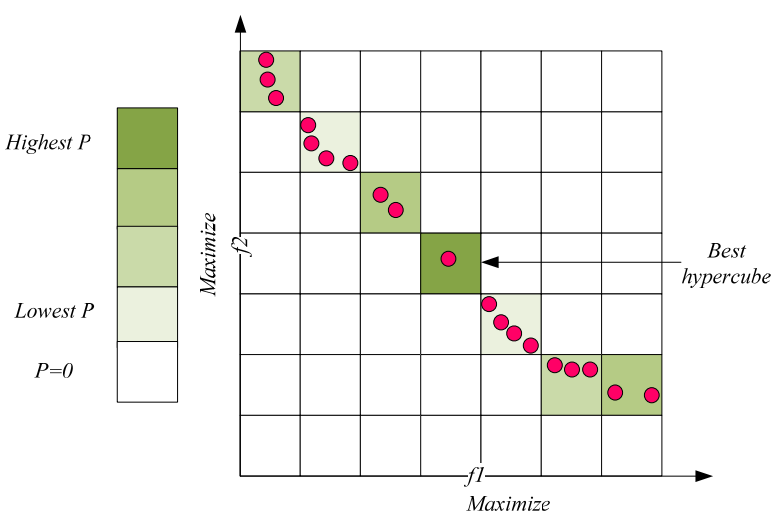

Figure 3: Conceptual model of the selection operator

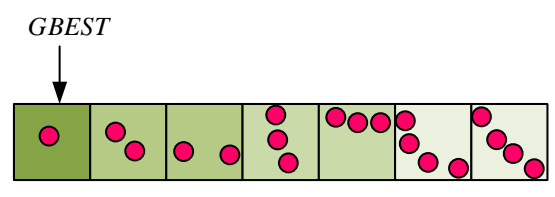

Figure 4: Selection of GBEST from different hypercubes

\subsection{1. $M O P S O$}

Handling multiple objectives using the PSO algorithm was proposed by Coello Coello et al. in 2004 $[33,34]$. MOPSO uses the same equation to update the particles in each iteration with slight changes as follows:

$v_{i}^{t+1}=w \cdot v_{i}^{t}+c_{1} \cdot r_{1} \cdot\left(P B E S T_{i}-X_{i}^{t}\right)-c_{2} \cdot r_{2} \cdot\left(G B E S T-X_{i}^{t}\right)$

$$
X_{i}^{t+1}=X_{i}^{t}+V_{i}^{t+1}
$$

where $t$ shows the current iteration, $i$ is the $i^{\text {th }}$ solution, $X_{i}^{t}$ indicates the position vector of $i^{\text {th }}$ in $t^{\text {th }}$ iteration, GBEST is one of the Pareto optimal solutions found sofar, and $P B E S T_{i}$ shows the best Pareto optimal solution that $i^{\text {th }}$ particle obtained so far.

It may be seen in the description of equations that the changes are applied to GBES T and PBEST. In a multiobjective search space there is no single best solution, so GBEST should be regularly updated with Pareto optimal solutions obtained so far. Therefore, MOPSO employs a repository (or archive) to store Pareto optimal solutions and a selector to assign them to the GBEST.

The repository is a storage to store Pareto optimal solutions with a limited size. To calculate the distribution of solutions around each Pareto optimal solution in the archive, Coello Coello et al. proposed a grid mechanism as shown in Fig. 3. This figure also shows a conceptual model of the selection operator.

It may be seen in Fig. 3 and 4 that solutions are selected from less populated regions. In fact, the probability for a solution to be the GBEST in each iteration is inversely proportional to its neighbourhood's distribution. The less number of solutions in the neighbourhood, the higher probability of being selected as the GBEST. This mechanism improves the change of enhancing the distribution of solutions in the repository.

For each particle, the PBSET is updated in every iteration as well. Each particle has its own storage to store the non-dominated PBEST. If the particle finds a solution that dominates the PBEST, MOPSO replaces it by the new solution. If the PBEST dominates the new solution, the PBEST remains intact for the next iteration. If both solutions are non-dominated compared to each other, one of them is chosen randomly (50\%). 
After updating all the solutions, the repository is updated as well. If there is any better solution outside the repository, it is transferred to or replaced by a dominated solution in the archive. If there is a non-dominated solution outside the archive, it is inserted into the archive as well. Since the size of repository is limited, it is frequently become full of solutions.An alternative mechanism of improving the distribution of solutions in the archive, solutions in crowded regions are discarded. Fig. 5 shows a conceptual example of this process.

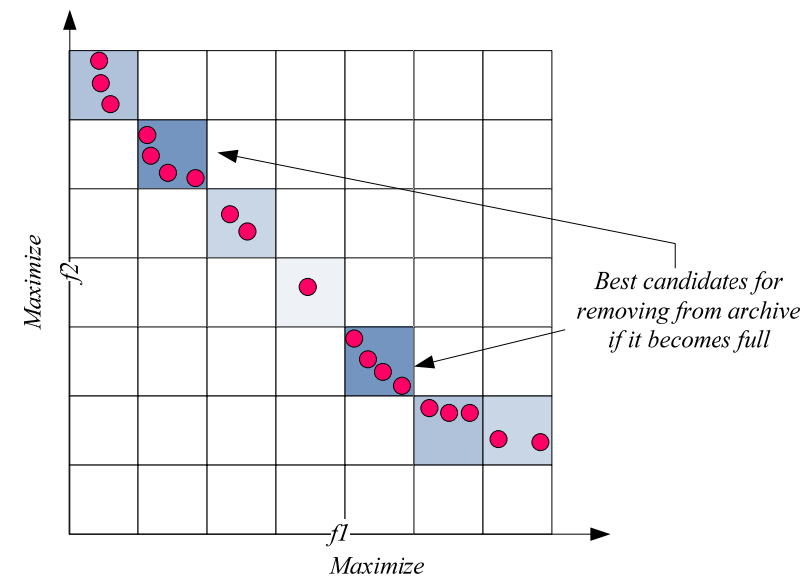

Figure 5: Updating mechanism of archive when the archive is full

In the standard MOPSO, all parameters of particles are changed with the same rate. Exploratory and exploitative mechanisms manipulate the particles similarly. This is not desired for problems with variables of different types. The next section proposes a novel mechanism to solve such problems for the fist time in the literature of hand posture estimation.

\section{MOPSO+EPD}

Evolutionary Population Dynamics is a natural phenomenon in evolution. It refers to the removal of the least fit individuals in a species which contributes to better adaptability to the environment over time [35, 36]. It is one of the key concepts in the Self Organizing Criticality theory [37]. According to the SOC theory, small mutations (perturbations) provide delicate balances in a balanced population without external force [38]. In the evolution of different species, it is observed that evolution also applies to the poor species. In this case, the total population of a species are affected by the removal of the poor individuals. The concept of this operator is illustrated in Fig. 6. An evolutionary algorithm equipped with this operator removes a set of worst solutions in

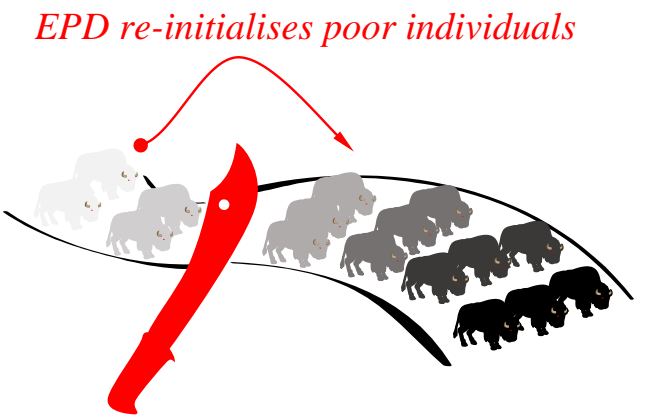

Figure 6: The concepts of Evolutionary Population Dynamics. Poor individuals perish, so their genes do not get involved in the creation of the next population. This is in contrast to the elitism, in which the best individuals have a higher chance of survival and participating in producing the next generation. To simulate this, poor solutions of an optimisation are discarded and replaced with random ones.

each generation and re-initialise them. Therefore, it behaves opposite to Genetic Algorithm (GA) [39], in which best solutions are selected to reproduce new solutions.

The main reason why EPD is potentially able to improve the performance of PSO and MOPSO is that it improves the median of the population by removing the worst individuals. The removal of worst individuals is the first step when using EPD in a population-based algorithm. According to Bak's theory [40], EPD (with small changes) is likely to help MOPSO to approach near-optimal solutions rapidly. Then the primary mechanisms of MOPSO search around those near-optimal solutions. In other words, they mutate or re-position the removed individuals around the best solutions.

Note that EPD is in contrast to the elitism operator in evolutionary algorithms. Elites are the best solutions and are maintained to participate in making the next population. In the EPD, however, the poor solutions are removed so they do not participate in the creation of the next population. This potentially minimises their undesirable values (variables) to be combined with other solutions. A 70\% elitism strategy guarantees that at least $70 \%$ of the population will be preserved unchanged to the next generation. A $30 \%$ EPD guarantees that $30 \%$ of the population will be removed/replaced - it does not guarantee that the remainder will be unchanged. Generally, an increasing proportion of unchanged population members increases the probability of convergence to local minima - there are less and less solutions in the population that have a chance to find a way out, by changing significantly.

The main motivation of applying EPD to MOPSO is to improve the exploration and local optima avoid- 


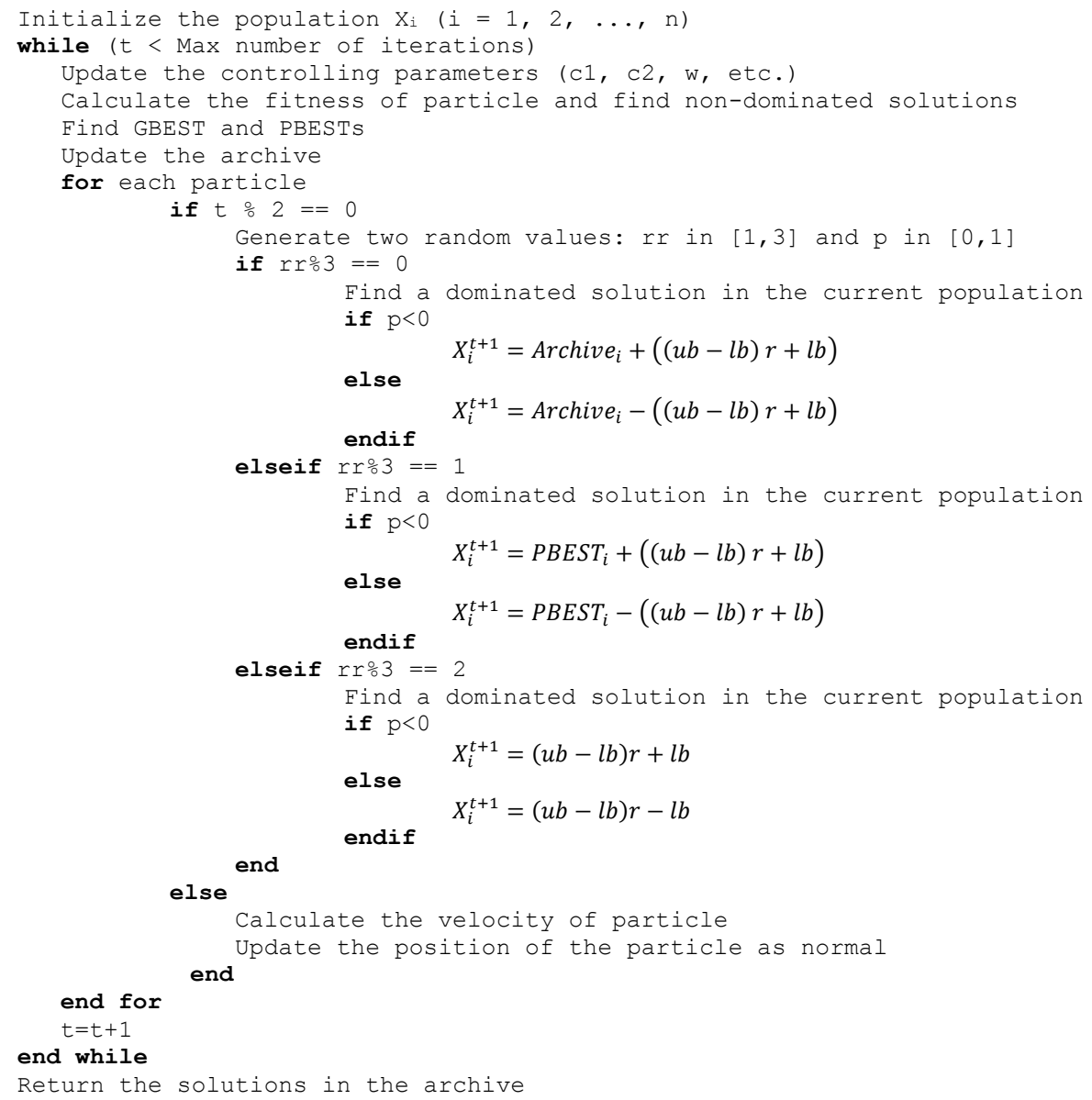

Figure 7: Pseudocode of the proposed PSO+EPD. Note that $r r \% 3$ returns the remainder of $r$ divided by three where $r r$ is a random integer in the interval of $[1,3]$. This gives $33 \%$ chance of choosing any of the three position updating equations.

ance. For the PSO [41] algorithm, it is easy to find worst solutions due to the existence of one objective. In MOPSO, however, multiple objectives are considered. Therefore, we have to find dominated solutions and reinitialise them. Also, there is no single GBEST solution in MOPSO, and there is an archive which stores the best non-dominated solutions obtained so far. Therefore, there should be a mechanism to select one of the solutions in the archive. To improve the probability of increasing the coverage of solutions, GBEST is selected from the low populated regions of the archive. This is done using a roulette wheel mechanism that gives a higher chance of selection to the solutions in the less population regions. The main equation to update the position of a particle using EPD is as follows:

$$
X_{i}^{t+1}=\operatorname{Archive~}_{i} \pm((u b-l b) \cdot r+l b)
$$

where $u b$ is the upper bound, $l b$ indicates the lower bound, $r$ is a random number generated using a uniform distribution in [0,1], and Archive ${ }_{i}$ indicates the $i^{\text {th }}$ solution in the archive selected by the roulette wheel.

In MOPSO, each solution has a non-dominated solution as the personal best solution found so-far. Therefore, the following equation is used to re-initialise 'bad' solutions around the PBEST S as well.

$$
X_{i}^{t+1}=P B E S T_{i} \pm((u b-l b) \cdot r+l b)
$$

where $u b$ is the upper bound, $l b$ indicates the lower bound, $r$ is a random number generated using a uniform distribution in $[0,1]$, and $P B E S T_{i}$ indicates the best solution obtained so-far by the $i^{\text {th }}$ particle.

With the two mechanisms proposed so far, the exploration can be improved significantly. To improve it 
even further, the following equation is used to relocate a solution to a completely random location in the search space.

$$
X_{i}^{t+1}=(u b-l b) \cdot r+l b
$$

where $u b$ is the upper bound, $l b$ indicates the lower bound, and $r$ is a random number generated using a uniform distribution in $[0,1]$.

Note that in all the above equations, \pm is done with $50 \%$ probability. For instance, Eq. 10 can be written as:

$$
X_{i}^{t+1}= \begin{cases}(u b-l b) \cdot r+l b, & \text { if } p \geq 0.5 \\ (u b-l b) \cdot r-l b, & \text { if } p<0.5\end{cases}
$$

where $p$ is a random number generated using a uniform distribution in $[0,1], u b$ is the upper bound, $l b$ indicates the lower bound, $r$ is a random number generated using a uniform distribution in $[0,1]$.

The rational behind the first two proposed rules is to improve the median of whole population and coverage of solutions in the archive in each iteration. However, the last rule re-positions the particles randomly around the search space, a very helpful mechanism to promote exploration and resolving local optima stagnation. The pseudo-code of the proposed algorithm is shown in Fig. 7. It may be seen that EDP is applied to every two iterations only. This can be changed depending on the level of exploration required for a given problem. Our initial investigation shows that applying EPD to every iteration returns poor results in most of the problem since it highly reduces the exploitation and accuracy of the solution obtained. We have tested applying EPD every 2,3,4, and 5 iterations and observed that the best results are achieved when applying EPD in every two iterations.

To see how the proposed MOPSO+EPD can be theoretically effective some remarks are:

1. MOPSO+EPD is able to avoid local optima better than MOPSO since the solutions are significantly changed in some iterations.

2. MOPSO+EPD removes the dominated solutions, so the best solutions in the current population do not mistakingly re-initialised.

3. Re-initializing the dominated solutions around non-dominated solutions in less population regions of archive improves the chance of enhancing the distribution of non-dominated solutions in the archive across all objectives.

4. Applying EPD to some of the iterations minimises degraded exploitation and convergence speed of MOPSO+EPD.
5. MOPSO+EPD benefits from the main features of MOPSO (derivative free, saving best solutions obtained so far, etc.) since there is no change in the main equations of MOPSO.

6. MOPSO+EPD is readily applicable to different problems since it considers problems as a black box.

7. MOPSO+EPD has a good chance of resolving local optima stagnation since there are three mechanisms to randomly relocate particles around the search space.

8. The computational complexity of MOPSO+EPD is similar to that of MOPSO.

9. EPD can be applied to some of the variables to fluctuate them more than others.

\section{Experimental results on test functions}

In this section, the performance of the proposed MOPSO+EPD is benchmarked on two sets of test functions: ZDT [42] and CEC2009 [43]. These two test suites provide a variety of challenging test functions to benchmark the performance of the proposed algorithm. The results of MOPSO+EPD are quantified with three performance metrics (discussed below) and compared with MOPSO, NSGA-II [44], and MOEA/D [45]. The initial parameters of these algorithms are given in Table 1. Note that we have used the most recent version of MOPSO to develop MOPSO+EPD and verify the results (the MOPSO algorithm in the tables).

\subsection{Results on ZDT test suite}

This section benchmarks the performances of the improved MOPSO on a set of multi-objective test functions. The test functions are extracted from the wellknown ZDT test suited [42]. Note MOPSO and NSGAII are used in the experiments to verify its performance as the most well-regarded multi-objective algorithm in the literature. The same number of function evaluations is used for all algorithms to provide a fair comparison. Since the true Pareto optimal front of the multiobjective test functions is known, the algorithms can be compared quantitatively. In the field of multi-objective optimisation, there is a large number of performance measures. The performance measure that is employed in this section to quantify the performance of algorithm and compared the results is Inverted Generational Distance (IGD). The mathematical formulation of this performance measure is defined as follows [46]:

$$
I G D=\frac{\sqrt{\sum_{i=1}^{n}\left(d_{i}\right)^{2}}}{n}
$$


Table 1: Initial values for the parameters of multi-objective algorithms when solving multi-objective test functions.

\begin{tabular}{|c|c|c|}
\hline Algorithm & Parameter & Value \\
\hline \multirow[t]{8}{*}{ MOPSO+EPD } & $\phi_{1}, \phi_{2}$ & 2.05 \\
\hline & $\phi$ & $\phi_{1}+\phi_{2}$ \\
\hline & $\omega=$ & $\frac{2}{\phi-2+\sqrt{\phi^{2}-4 \phi}}$ \\
\hline & $c_{1}$ & $\phi_{1}$ \\
\hline & $c_{2}$ & $\phi_{2}$ \\
\hline & $\alpha$ & 0.1 \\
\hline & $\beta$ & 4 \\
\hline & nGrid & 10 \\
\hline \multirow[t]{8}{*}{ MOPSO } & $\phi_{1}, \phi_{2}$ & 2.05 \\
\hline & $\phi$ & $\phi_{1}+\phi_{2}$ \\
\hline & $\omega$ & $\frac{2}{\phi-2+\sqrt{\phi^{2}-4 \phi}}$ \\
\hline & $c_{1}$ & $\phi_{1}$ \\
\hline & $c_{2}$ & $\phi_{2}$ \\
\hline & $\alpha$ & 0.1 \\
\hline & $\beta$ & 4 \\
\hline & nGrid & 10 \\
\hline \multirow[t]{2}{*}{ NSGA-II } & Mutation probability & 0.2 \\
\hline & Crossover rate & 0.8 \\
\hline \multirow[t]{5}{*}{ MOEA/D } & Sub-problems (N) & 100 \\
\hline & $\mathrm{T}$ & 10 \\
\hline & $\begin{array}{l}n_{r} \\
\delta=0.9\end{array}$ & 1 \\
\hline & $\mathrm{CR}, \mathrm{F}$ & 0.5 \\
\hline & $\eta$ & 30 \\
\hline
\end{tabular}

Table 2: Results of MOPSO+EPD, MOPSO, and NSGA-II on ZDT test functions

\begin{tabular}{llllll}
\hline Algorithm & ZDT1 & & & & \\
\hline & Ave & Std. & Median & Best & Worst \\
\hline MOPSO+EPD & 0.00326 & 0.00329 & 0.002240 & 0.00055 & 0.00850 \\
MOPSO & 0.00422 & 0.00310 & 0.0037 & 0.0015 & 0.0101 \\
NSGA-II & 0.05988 & 0.00544 & 0.0574 & 0.0546 & 0.0702 \\
\hline & ZDT2 & & & & \\
\hline MOPSO+EPD & 0.00068 & 0.00014 & 0.00073 & 0.00050 & 0.00084 \\
MOPSO & 0.00156 & 0.00017 & 0.00170 & 0.00130 & 0.00170 \\
NSGA-II & 0.13972 & 0.02626 & 0.12580 & 0.11480 & 0.18340 \\
\hline & ZDT3 & & & & \\
\hline MOPSO+EPD & 0.03518 & 0.00217 & 0.03610 & 0.03130 & 0.03640 \\
MOPSO & 0.03782 & 0.00630 & 0.03620 & 0.03080 & 0.04970 \\
NSGA-II & 0.04166 & 0.00807 & 0.04030 & 0.03150 & 0.05570 \\
\hline
\end{tabular}

where $n$ is the number of true Pareto optimal solutions and $d_{i}$ indicates the Euclidean distance between the $i$ th true Pareto optimal solution and the closest Pareto optimal solution obtained in the reference set.

Each algorithm was run 30 times and the average results of IGD is provided in Table 2. This table shows that the MOPSO+EPD algorithm provides very competitive results compared to other algorithms. Both MOPSO+EPD and MOPSO outperform the NSGA-II algorithm. This is due to a better exploitation of the algorithms since slight changes in the solutions using the EPD operator makes minor changes in solutions and promote local search. The superiority is very significant in the first four test cases. Comparing the results of MOPSO+EPD and MOPSO, it is evident that this algorithm outperformed MOPSO and NSGA-II significantly. We conducted Wilcoxon rank-sum test at 5\% significance level. The search landscape of these two test functions are highly multi-modal. These results show that the EPD operator improved the exploration and local (false) front avoidance of the MOPSO algorithm. Obviously, if we apply EPD in every iteration, that might degrade the exploitation and convergence of MOPSO. The proposed mechanism in which EPD is applied every two iterations prevents this issue as confirmed by the results of ZDT1 and ZDT2 as test functions with unimodal landscapes.

\subsection{Results on composite (CEC2009) test functions}

To further investigate the performance of the proposed MOPSO+EPD algorithm, one of the most challenging test suites in the literature called CEC2009 has been employed. The mathematical equations of these algorithms can be found in [43]. These test functions mimic the difficulties of a search space: a large number of local fronts, constraints, isolated front, front on the boundary, etc. Due to the difficulty of these test functions, 100 particles and 3000 iterations are used in this subsection. The results of the proposed MOPSO+EPD are compared with those of MOPSO and Multi-Objective Evolutionary Algorithm based on Decomposition (MOEA/D) [45]. To quantify the results, IGD, Metric of Spacing (SP) [47], and Maximum Spread (MS) [48]. The last two metrics quantify the coverage of algorithms and are defined as follows:

$$
S P=\sqrt{\frac{1}{n-1} \sum_{i=1}^{n}\left(\bar{d}-d_{i}\right)^{2}}
$$

where $\bar{d}$ is the average of all $d_{i}, n$ is the number of Pareto optimal solutions obtained, and $d_{i}=\min _{j}\left(\mid f_{1}^{i}(\vec{x})\right.$ $\left.f_{1}^{j}(\vec{x})|+| f_{2}^{i}(\vec{x})-f_{2}^{j}(\vec{x}) \mid\right)$ for all $i, k=1,2,3, \ldots, n$. 


$$
M S=\sqrt{\sum_{i=1}^{o} \max \left(d\left(a_{i}, b_{i}\right)\right)}
$$

where $d$ is a function to calculate the Euclidean distance, $a_{i}$ is the maximum value in the $i^{\text {th }}$ objective, $b_{i}$ is the minimum in the $i^{\text {th }}$ objective, and o is the number of objectives.

The quantitative results collected using the IGD metric are presented in Table 3. Note that to compare the quantitative results of each run and decide on the significance of the results, the Wilcoxon statistical test is performed at $5 \%$ significance level, and the p-values are reported in Tables 5 to 8 . For the statistical test, the best algorithm in each test function is chosen and compared with other algorithms independently. For example, if the best algorithm is MOPSO+EPD, a pairwise comparison is made between MOPSO+EPD/PSO, MOPSO+EPD/MOPSO, and MOPSO+EPD/MOEAD. Note that since the best algorithm cannot be compared with itself, N/A is written for the best algorithm in each function which stands for Not Applicable.

It may be seen that MOPSO+EPD outperforms both MOPSO and MOEA/D on UF1, UF6, and UF7. This algorithm also provides better results that MPSO on all the three-objective test functions (UF8, UF9, and UF10). For the rest of test functions MOPSO+EPD shows very competitive results. The IGD performance indication mostly measures convergence of multi-objective algorithms and defines how well they perform in finding an accurate approximation of the true Pareto optimal solutions. Therefore, these results show that MOPSO+EPD benefits from high convergence. This can be seen in the best Pareto optimal front obtained by algorithms in Figs. 8 and 9. These figure show that the Pareto optimal solutions estimated by MOPSO+EPD tend to be closer to the true Pareto optimal front compared to MOPSO and MOEA/D.

On the other hand, SP and MS quantifies coverage of multi-objective algorithms. Note that high values for MS and low values for SP show more uniform distribution of estimated Pareto optimal solutions. The results collected using SP and MS are given in Table 5 to 8 respectively. It may be seen that the MOPSO+EPD technique is superior on almost half of the test functions. This shows that the coverage of solutions obtained by this algorithm is of high distribution across all objective on the test problems. This can be seen in Fig. 8 and 9 as well. It is observed that the solutions found by MOPSO+EPD are well distributed on the majority of test problems.

To sum up, the results of this section show that the
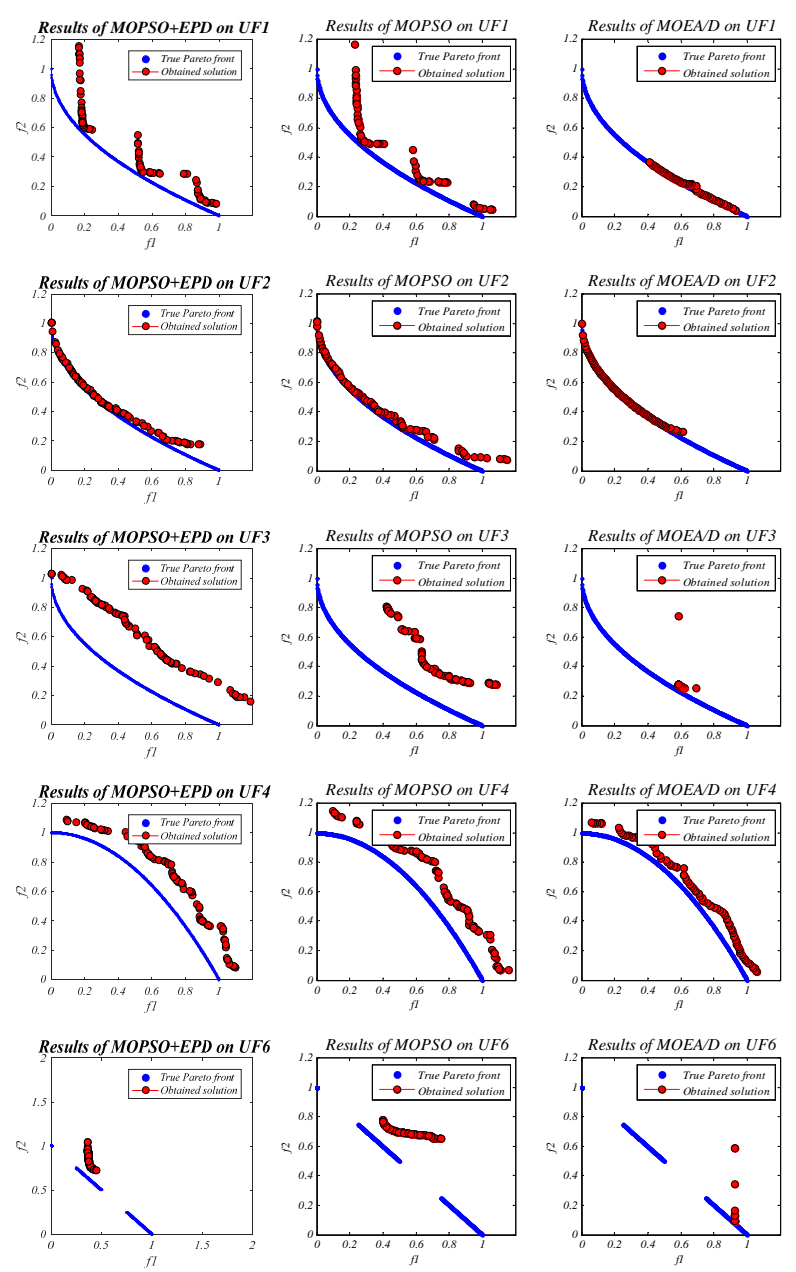

Figure 8: Best Pareto optimal front estimated by the algorithms on CEC2009 test functions 
This paper has been published in Knowledge-Based Systems.

Table 3: Statistical results for IGD on UF1 to UF7. The results for the average are represented in the form of ave( pval) where pval indicates the p-value obtained from the Wilcoxon ranksum test.

\begin{tabular}{|c|c|c|c|c|c|c|}
\hline & UF1 & & & UF2 & & \\
\hline IGD & MOPSO+EPD & MOPSO & MOEA/D & MOPSO+EPD & MOPSO & MOEA/D \\
\hline Average & $0.129(\mathrm{~N} / \mathrm{A})$ & $0.136(0.0468)$ & $0.120(0.0312)$ & $0.063(0.2730)$ & $0.060(\mathrm{~N} / \mathrm{A})$ & $0.122(0.0017)$ \\
\hline Median & 0.124 & 0.131 & 0.113 & 0.060 & 0.048 & 0.120 \\
\hline STD. Dev. & 0.026 & 0.042 & 0.039 & 0.017 & 0.028 & 0.011 \\
\hline \multirow[t]{2}{*}{ Best } & 0.088 & 0.090 & 0.057 & 0.046 & 0.037 & 0.105 \\
\hline & UF3 & & & UF4 & & \\
\hline IGD & MOPSO+EPD & MOPSO & MOEA/D & MOPSO+EPD & MOPSO & MOEA/D \\
\hline Average & $0.292(0.9097)$ & $0.314(0.3074)$ & 0.289 (N/A) & $0.128(0.0001)$ & $0.136(0.0001)$ & $0.068(\mathrm{~N} / \mathrm{A})$ \\
\hline Median & 0.289 & 0.308 & 0.289 & 0.128 & 0.134 & 0.068 \\
\hline STD. Dev. & 0.047 & 0.045 & 0.016 & 0.007 & 0.007 & 0.002 \\
\hline \multirow[t]{2}{*}{ Best } & 0.239 & 0.256 & 0.263 & 0.119 & 0.127 & 0.065 \\
\hline & UF5 & & & UF6 & & \\
\hline IGD & MOPSO+EPD & MOPSO & MOEA/D & MOPSO+EPD & MOPSO & MOEA/D \\
\hline Average & $2.099(0.0001)$ & $2.202(0.0002)$ & $1.291(\mathrm{~N} / \mathrm{A})$ & 0.491 (N/A) & $0.648(0.0121)$ & $0.688(0.001)$ \\
\hline Median & 2.008 & 2.126 & 1.338 & 0.421 & 0.551 & 0.698 \\
\hline STD. Dev. & 0.380 & 0.553 & 0.135 & 0.119 & 0.266 & 0.055 \\
\hline \multirow[t]{2}{*}{ Best } & 1.679 & 1.465 & 1.123 & 0.353 & 0.379 & 0.552 \\
\hline & UF7 & & & & & \\
\hline IGD & MOPSO+EPD & MOPSO & MOEA/D & & & \\
\hline Average & $0.295(\mathrm{~N} / \mathrm{A})$ & $0.354(0.0420)$ & $0.506(0.0239)$ & & & \\
\hline Median & 0.333 & 0.387 & 0.516 & & & \\
\hline STD. Dev. & 0.189 & 0.204 & 0.151 & & & \\
\hline Best & 0.060 & 0.054 & 0.029 & & & \\
\hline
\end{tabular}

Table 4: Statistical results for IGD on UF8 to UF10. The results for the average are represented in the form of ave( $p v a l$ ) where $p v a l$ indicates the p-value obtained from the Wilcoxon ranksum test.

\begin{tabular}{lllllll}
\hline \multicolumn{2}{r}{} & UF8 & & UF9 & & UF10 \\
\hline IGD & MOPSO+EPD & MOPSO & MOPSO+EPD & MOPSO & MOPSO+EPD & MOPSO \\
\hline Average & 0.417 (N/A) & $0.537(0.0389)$ & 0.454 (N/A) & $0.489(0.0425)$ & 1.550 (N/A) & $1.637(0.0270)$ \\
Median & 0.449 & 0.536 & 0.405 & 0.415 & 1.520 & 1.592 \\
STD. Dev. & 0.142 & 0.183 & 0.139 & 0.144 & 0.359 & 0.299 \\
Best & 0.238 & 0.255 & 0.311 & 0.334 & 1.031 & 1.220 \\
\hline
\end{tabular}

Table 5: Statistical results for SP on UF1 to UF7. The results for the average are represented in the form of $a v e(p v a l)$ where $p v a l$ indicates the p-value obtained from the Wilcoxon ranksum test.

\begin{tabular}{|c|c|c|c|c|c|c|}
\hline & UF1 & & & UF2 & & \\
\hline SP & MOPSO+EPD & MOPSO & MOEA/D & MOPSO+EPD & MOPSO & MOEA/D \\
\hline Average & $0.008(0.0004)$ & $0.009(0.0001)$ & $0.004(\mathrm{~N} / \mathrm{A})$ & $0.008(\mathrm{~N} / \mathrm{A})$ & $0.008(0.3074)$ & $0.009(0.0091)$ \\
\hline Median & 0.008 & 0.009 & 0.003 & 0.007 & 0.008 & 0.009 \\
\hline STD. Dev. & 0.002 & 0.002 & 0.002 & 0.001 & 0.002 & 0.001 \\
\hline \multirow[t]{2}{*}{ Best } & 0.006 & 0.007 & 0.002 & 0.006 & 0.006 & 0.008 \\
\hline & UF3 & & & UF4 & & \\
\hline SP & MOPSO+EPD & MOPSO & MOEA/D & MOPSO+EPD & MOPSO & MOEA/D \\
\hline Average & $0.007(\mathrm{~N} / \mathrm{A})$ & $0.007(0.5205)$ & $0.027(0.0257)$ & $0.007(0.9775)$ & $0.007(\mathrm{~N} / \mathrm{A})$ & $0.007(0.9775)$ \\
\hline Median & 0.007 & 0.007 & 0.025 & 0.007 & 0.007 & 0.007 \\
\hline STD. Dev. & 0.001 & 0.002 & 0.021 & 0.001 & 0.001 & 0.001 \\
\hline \multirow[t]{2}{*}{ Best } & 0.005 & 0.006 & 0.019 & 0.005 & 0.005 & 0.005 \\
\hline & UF5 & & & UF6 & & \\
\hline SP & MOPSO+EPD & MOPSO & MOEA/D & MOPSO+EPD & MOPSO & MOEA/D \\
\hline Average & $0.005(0.0091)$ & $0.008(0.0756)$ & $0.003(\mathrm{~N} / \mathrm{A})$ & $0.007(0.3051)$ & $0.021(0.0206)$ & $0.006(\mathrm{~N} / \mathrm{A})$ \\
\hline Median & 0.005 & 0.005 & 0.000 & 0.005 & 0.012 & 0.000 \\
\hline STD. Dev. & 0.007 & 0.004 & 0.006 & 0.009 & 0.033 & 0.013 \\
\hline \multirow[t]{2}{*}{ Best } & 0.001 & 0.000 & 0.000 & 0.001 & 0.002 & 0.000 \\
\hline & UF7 & & & & & \\
\hline SP & MOPSO+EPD & MOPSO & MOEA/D & & & \\
\hline Average & $0.007(0.1619)$ & $0.007(0.1619)$ & $0.004(\mathrm{~N} / \mathrm{A})$ & & & \\
\hline Median & 0.007 & 0.007 & 0.003 & & & \\
\hline STD. Dev. & 0.003 & 0.003 & 0.002 & & & \\
\hline Best & 0.004 & 0.003 & 0.002 & & & \\
\hline
\end{tabular}


This paper has been published in Knowledge-Based Systems.

Table 6: Statistical results for SP on UF8 to UF10. The results for the average are represented in the form of ave( pval) where pval indicates the p-value obtained from the Wilcoxon ranksum test.

\begin{tabular}{lllllll}
\hline & UF8 & & UF9 & & UF10 & \\
\hline SP & MOPSO+EPD & MOPSO & MOPSO+EPD & MOPSO & MOPSO+EPD & MOPSO \\
\hline Average & 0.026 (N/A) & $0.027(0.0498)$ & $0.023(\mathrm{~N} / \mathrm{A})$ & $0.023(0.9531)$ & 0.019 (N/A) & $0.020(0.0391)$ \\
Median & 0.025 & 0.026 & 0.023 & 0.023 & 0.019 & 0.021 \\
STD. Dev. & 0.005 & 0.008 & 0.004 & 0.005 & 0.003 & 0.003 \\
Best & 0.011 & 0.015 & 0.017 & 0.017 & 0.013 & 0.015 \\
\hline
\end{tabular}

Table 7: Statistical results for MS on UF1 to UF7. The results for the average are represented in the form of ave( pval) where pval indicates the p-value obtained from the Wilcoxon ranksum test.

\begin{tabular}{|c|c|c|c|c|c|c|}
\hline & UF1 & & & UF2 & & \\
\hline MS & MOPSO+EPD & MOPSO & MOEA/D & MOPSO+EPD & MOPSO & MOEA/D \\
\hline Average & $1.263(0.7337)$ & $1.267(\mathrm{~N} / \mathrm{A})$ & $0.276(0.0312)$ & $0.840(0.1619)$ & $0.864(\mathrm{~N} / \mathrm{A})$ & $0.648(0.0017)$ \\
\hline Median & 1.314 & 1.120 & 0.325 & 0.846 & 0.868 & 0.651 \\
\hline STD. Dev. & 0.469 & 0.690 & 0.325 & 0.115 & 0.122 & 0.016 \\
\hline \multirow[t]{2}{*}{ Best } & 1.940 & 2.885 & 0.434 & 1.050 & 1.120 & 0.674 \\
\hline & UF3 & & & UF4 & & \\
\hline MS & MOPSO+EPD & MOPSO & MOEA/D & MOPSO+EPD & MOPSO & MOEA/D \\
\hline Average & $1.406(\mathrm{~N} / \mathrm{A})$ & $1.391(0.0427)$ & $0.159(0.0001)$ & $1.025(0.0055)$ & $1.037(\mathrm{~N} / \mathrm{A})$ & $1.003(0.0001)$ \\
\hline Median & 1.303 & 1.177 & 0.123 & 1.028 & 1.038 & 1.003 \\
\hline STD. Dev. & 0.402 & 0.740 & 0.132 & 0.018 & 0.022 & 0.023 \\
\hline \multirow[t]{2}{*}{ Best } & 2.224 & 3.306 & 0.414 & 1.045 & 1.073 & 1.041 \\
\hline & UF5 & & & UF6 & & \\
\hline MS & MOPSO+EPD & MOPSO & MOEA/D & MOPSO+EPD & MOPSO & MOEA/D \\
\hline Average & $1.270(\mathrm{~N} / \mathrm{A})$ & $1.065(0.0113)$ & $0.032(0.0041)$ & $0.606(0.2730)$ & $1.516(\mathrm{~N} / \mathrm{A})$ & $0.045(0.009)$ \\
\hline Median & 1.231 & 1.032 & 0.006 & 0.268 & 1.424 & 0.000 \\
\hline STD. Dev. & 0.911 & 0.966 & 0.044 & 0.726 & 1.005 & 0.110 \\
\hline \multirow[t]{2}{*}{ Best } & 3.363 & 2.404 & 0.116 & 2.084 & 2.683 & 0.348 \\
\hline & UF7 & & & & & \\
\hline MS & MOPSO+EPD & MOPSO & MOEA/D & & & \\
\hline Average & $0.786(0.6775)$ & $1.011(\mathrm{~N} / \mathrm{A})$ & $0.218(0.2122)$ & & & \\
\hline Median & 0.835 & 0.998 & 0.159 & & & \\
\hline STD. Dev. & 0.377 & 0.731 & 0.287 & & & \\
\hline Best & 1.275 & 2.311 & 0.983 & & & \\
\hline
\end{tabular}

Table 8: Statistical results for MS on UF8 to UF10. The results for the average are represented in the form of ave( pval) where $p v a l$ indicates the p-value obtained from the Wilcoxon ranksum test.

\begin{tabular}{lllllll}
\hline \multicolumn{2}{c}{ UF8 } & \multicolumn{3}{c}{ UF9 } & UF10 & \\
\hline MS & MOPSO+EPD & MOPSO & MOPSO+EPD & MOPSO & MOPSO+EPD & MOPSO \\
\hline Average & $4.065(\mathrm{~N} / \mathrm{A})$ & $3.425(0.0472)$ & $3.838(0.7337)$ & $4.326(\mathrm{~N} / \mathrm{A})$ & $2.913(0.5205)$ & $3.076(\mathrm{~N} / \mathrm{A})$ \\
Median & 3.134 & 3.680 & 4.148 & 3.953 & 2.579 & 3.268 \\
STD. Dev. & 2.100 & 1.315 & 1.756 & 1.827 & 1.136 & 0.875 \\
Best & 8.456 & 5.265 & 7.079 & 6.911 & 5.080 & 4.263 \\
\hline
\end{tabular}




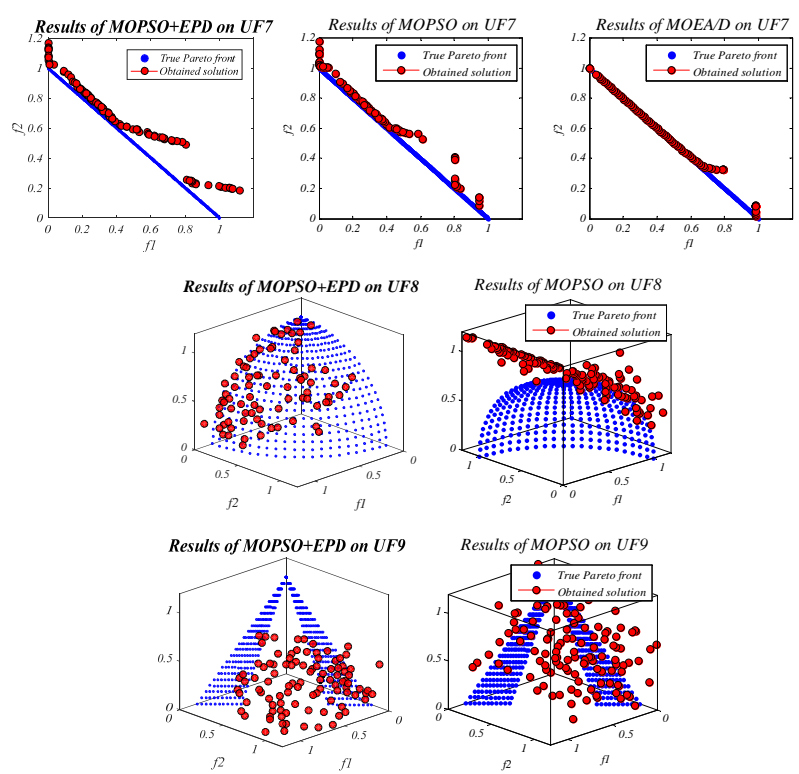

Figure 9: Best Pareto optimal front estimated by the algorithms on CEC2009 test functions

MOPSO+EDP is able to outperform MOPSO, NSGAII, and MOEA/D on most of the test functions. The results of this algorithm are very competitive on the rest of functions, which show the merits of this algorithm compared to the well-regarded algorithms employed. The superiority is due to the higher local optima (front) avoidance of this algorithm using the concept of EPD. Therefore, it has the potential to be used for the problem with a lot of local optima. The next section investigates the efficiency of this algorithm in the field of hand posture estimation for the first time in the literature.

\section{Hand shape/posture estimation}

\subsection{Two-objective formulation of the hand posture es- timation problem}

The problem of estimating hand model can be formulated as a two-objective problem to minimise the discrepancy and minimise the number of points in the point cloud. These two objectives are in conflict, meaning that reducing the number of points will increase the discrepancy and error rate. In order to get a better estimation, we need a large number of points in the point cloud. As discussed in the literature review, addressing these two objectives simultaneously have not been investigated in the literature. Therefore, this section first formulates the problem as a multi-objective problem and then solve it with MOPSO+EPD.
According to the definition of multi-objective optimisation, there is no one single solution for such problems, but this depends on the problem. For a problem with single-objective, there is one global optimum since there is one criterion. For instance, hand pose estimation considering discrepancy will result in a set of parameters with minimum discrepancy (or multiple designs with equal discrepancy in case the search space is multi-modal). However, there is more than one solution when considering multiple objectives. The set of all the optimal solutions (non-dominated solutions) is called Pareto optimal set and their corresponding projection in the objective space is called Pareto optimal front. The problem of hand posture estimation can be formulated as a two-objective minimisation problem as follows:

$$
\begin{gathered}
\text { Minimise : } E(\vec{x}) \\
\text { Minimise }: n
\end{gathered}
$$

where $n$ is the number of points in the point cloud and $\vec{x}$ includes 27 variables defining the number of points on the hand shape (1), the angle of each joint depending on the number of DoF (20), location of wrist in the search space (3), and the orientation of hand (3).

With this problem formulation, a multi-objective algorithm is able to estimate the Pareto optimal front. It should be noted that the number of points is the second objective. However, it should be parametrised to be defined by the multi-objective optimiser as well. This is illustrated in Fig. 10. This figure shows that the parameters vector consists of 27 parameters for this twoobjective problem. The first six parameters define the global features of the 3D model (translation and rotation). The remaining 21 parameters indicate the local features: 20 for defining the skeleton (each joint degree of freedom) and one for the number of points extracted from the 3D model.

It should be noted that the last parameter in the vector $(n)$ shows the number of points in the point cloud and the reference point cloud extracted from the depth image as well. This is because there should be one associated point in the reference point cloud for every point extracted from the 3D hand model. The process of calculating the objective functions for the two-objective problem is illustrated in Fig. 11. It may be seen in this figure that the first objective is the average of the distance between all points in the point cloud extracted from the 3D model and their corresponding closest point in the reference point cloud be extracted from the depth image. The second objective is a parameter itself which defines the number of points in both point clouds. 


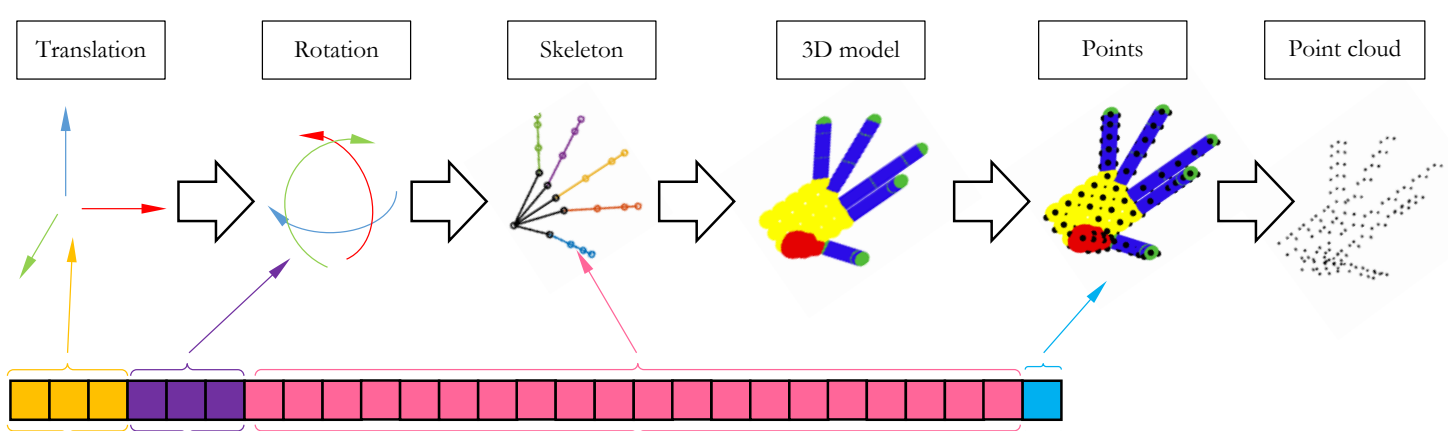

Figure 10: Proposed vector for hand model optimisation. There are 27 parameters in the parameters vector: translation (3), rotation (3), skeleton (20), and number of points on the 3D model (1)

\subsection{Experimental setup}

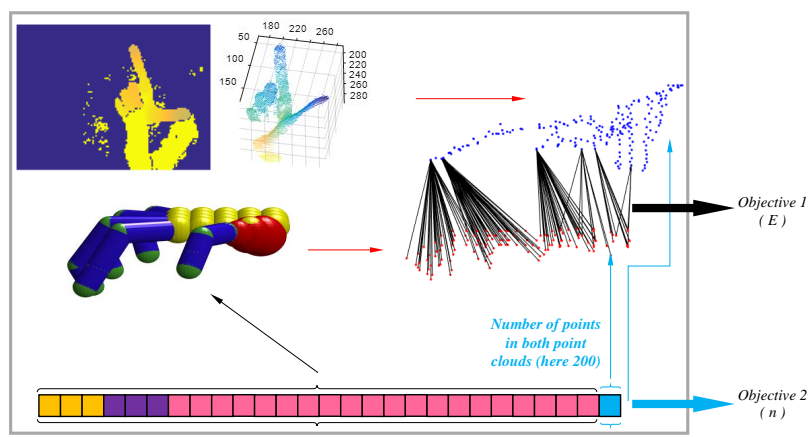

Figure 11: The process of calculating both objectives $(E$ and $n)$. Note that $n$ defines the number of points in the points clouds extracted from both 3D hand model and depth image

Table 9: Initial values for the parameters of MOPSO+EPD and MOPSO when solving hand postures

\begin{tabular}{ll}
\hline Parameter & Value \\
\hline$\phi_{1}, \phi_{2}$ & 2.05 \\
$\phi$ & $\phi_{1}+\phi_{2}$ \\
$\omega=$ & $\frac{2}{\phi-2+\sqrt{\phi^{2}-4 \phi}}$ \\
$c_{1}$ & $\phi_{1}$ \\
$c_{2}$ & $\phi_{2}$ \\
$\alpha$ & 0.1 \\
$\beta$ & 4 \\
nGrid & 10 \\
Number of particles & 60 \\
Number of iterations & 300 \\
\hline
\end{tabular}

The main constraints in this problem are the collisions between fingers or fingers/palm. The rest of constraints are on the range of parameters. To handle constraints, a death penalty function [49] is used, in which solutions will be assigned with large objective values in case of violating any of the constraints (collision between fingers and/or palm) at any levels. Although a better constraint handling might improve the performance, the main objective of this section is to estimate the Pareto optimal front for two-objective problem and provide relevant comparisons. To verify the results, MOPSO+EPD is compared to MOPSO. Note that MOPSO+EPD applies EPD to all parameters except translation and rotation. This is where we require MOPSO+EPD to assign priority to the variables. The priority of the first six variables are of the lowers without applying the EPD, whereas the rest of parameters are changed with a similar priority using EPD. For the second objective, number of points (n), a set of discrete values are chosen $(25,50,100,150, \ldots)$. This is because there will be no major difference between minor changes in the number of points in the point cloud.

A set of 50 postures have been collected from five datasets in the literature as shown in Fig. 23 in the appendix. The results have been collected and presented qualitatively. It is not possible to provide quantitative results since the true Pareto optimal front is unknown for this problem, so we cannot measure how close an estimated front is. However, with enough number of particles, iterations, and runs we can make sure that the Pareto optimal front obtained is close to the true front. After all, the initial parameters of MOPSO+EPD and MOPSO are given in Table 9. 

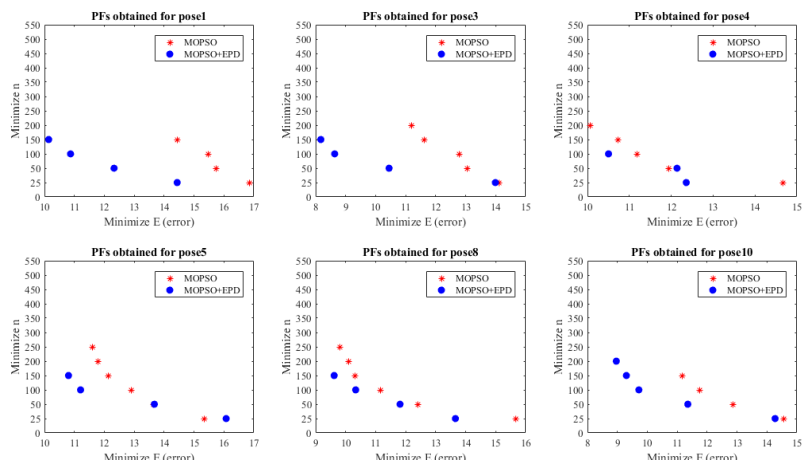

Figure 12: Comparison of MOPSO and MOPSO+EPD on the postures in the first dataset

\subsection{Benchmarking MOPSO+EPD on hand posture datasets without initialization}

The first experiment is on the efficiency of the proposed MOPSO+EPD when solving hand posture estimation problems considering two-objectives. This algorithm is run 30 times on each hand posture. The range of the second objective is $[25,550]$. Due to the large number of case studies in this paper, all the Pareto optimal fronts obtained are provided in the appendix (see Fig. 24 to Fig. 28). This section presents some of them that well represent all the patterns.

To qualitatively compare the results, the best Pareto optimal front for each hand model during 30 runs are illustrated in Fig. 12, 13, and 14. This figure shows that both MOPSO+EPD and MOPSO found four Pareto optimal solutions. However, the solutions obtained by MOPSO+EPD are distributed higher along the first objective $(E)$. Also, the solutions of MOPSO+EPD dominate those found by MOPSO. In Pose3, a similar pattern can be observed: MOPSO+EPD tends to outperform MOPSO. However, the right-most solutions obtained along the first objective are non-dominated with respect to each other.

It is interesting that the Pareto optimal front estimated by both algorithms are different for Pose4. In this pose, MOPSO finds a more distributed set of solutions. Despite this fact, the solutions are mostly dominated by a couple of solutions found by MOPSO+EPD. The behaviour of algorithm on Pose5 is similar to that on Pose3. PF obtained by MOPSO+EPD dominates that determined by MOPSO. However, the distribution of solutions for both algorithms are high and nearly uniform. It is worth noting that one of the solutions in PF od MOPSO dominates one of the solutions in MOPSO+EPD.

A similar pattern can be seen in Pose8, in which
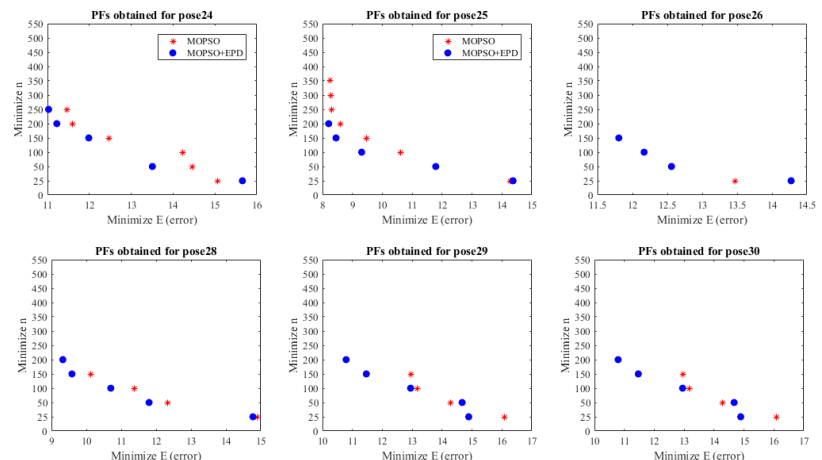

Figure 13: Comparison of MOPSO and MOPSO+EPD on the postures in the second dataset

MOPSO+EPD dominates most of the solutions in the $\mathrm{PF}$ of MOPSO. However, the distribution of solutions for the MOPSO algorithm is better. Regarding the distribution of solutions, an opposite pattern is observed in Pose10, in which the distribution of solutions obtained by MOPSO+EPD is significantly better than the solutions of MOPSO. All the solutions of MOPSO are dominated by MOPSO+EPD as well.

Pareto optimal solutions on Pose24 to Pose30 are visualised in Fig. 14. In Pose24, both algorithms found five Pareto optimal solutions. The distribution of solutions for MOPSO+EPD is high and they all dominate the solutions found by the MOPSO algorithm. The Pareto optimal fronts of algorithms in Pose25 is interesting. The solutions obtained by MOPSO gravitates towards the left hand side of the figure, while the solutions found by MOPSO+EPD are uniformly distributed along both objectives. All solutions of MOPSO+EPD are non-dominated compared to the solutions of MOPSO.

The MOPSO algorithm shows the poorest performance on Pose26. This algorithm found only one solution. In contrast, the performance of MOPSO+EDP on this pose is consistent to those on other poses: the distribution is high and they are all non-dominated. Although MOPSO found one solution for this pose, the single solution is non-dominated compared to the solutions found by MOPSO+EPD. Both algorithm show very competitive distributions when estimating Pose28. Despite this fact, once more, MOPSO+EPD outperforms the MOPSO algorithm significantly since most of the solutions dominate those found by MOPSO.

In Pose 30, the results of both algorithms are different from what was seen in the previous case studies. Inspecting the last two sub-figures in Fig. 13, the solutions obtained by both algorithms are not uniformly distributed along the second objective $(n)$. One of the 

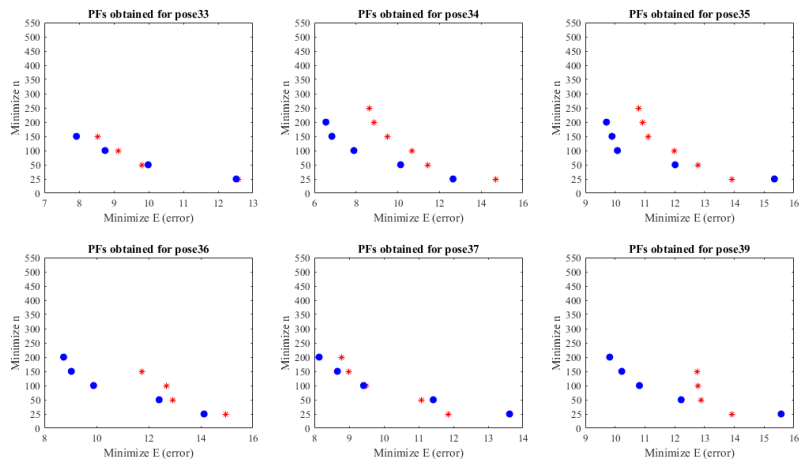

Figure 14: Comparison of MOPSO and MOPSO+EPD on the postures in the third dataset

solutions obtained by MOPSO dominates the associated solution in the Pareto front found by MOPSO. The middle solutions (along $E$ ) of both algorithms are nondominated with respect to each other as well. Despite all these facts, the better performance of MOPSO+EPD is evident.

The rest of results are illustrated in Fig. 14. In may be observed in this figure that the MOPSO+EPD algorithm outperforms the MOPSO algorithm in terms of both convergence and coverage when estimating Pose 33. The distance between solutions of both algorithms are not significant, showing that the MOPSO is competitive.

In contrary, the distance between solutions obtained by both algorithms in Pose34 is significant. It is evident in the second sub-figure that the solutions obtained by MOPSO+EPD significantly dominates those of MOPSO. The distribution of solutions found by MOPSO+EPD is better as well. A similar pattern can be seen in the Pareto optimal fronts for Pose 35, in which most of the solutions found by MOPSO+EPD are better than those of MOPSO. In this pose, however, the distribution of MOPSO seems to be more uniform.

The distribution of solutions estimated by MOPSO+EPD in Pose36 and Pose39 are substantially better than the solutions of MOPSO. All solutions of MOPSO are dominated by those of MOPSO+EPD. The MOPSO algorithm shows the most competitive results when estimating Pose37. The Pareto optimal fronts found by both algorithms in Fig. 14 show that the results are very competitive. Almost, $50 \%$ of solutions are non-dominated with respect to each other. However, the distribution of solutions found by MOPSO+EPD is higher than MOPSO.

\subsection{Finding a good range for $n$ and benchmarking $M O P S O+E P D$ on hand posture datasets with ini- tialization}

In the previous experiment the range of number of points in the point cloud was in $[25,550]$. It was observed that increasing the number of points does not necessarily decrease the error rate. To show this in details, a new experiment is conducted to use MOPSO+EPD and require this algorithm to vary the number of points. The range of points in the point cloud was relaxed in this experiment, meaning that it can be changed from 25 to 1500 . The MOPSO+EPD algorithm is run 30 times and the best Pareto optimal set obtained are illustrated in Fig. 15.

This figure shows that the best range for the number of points is between 25 to 500 . It may be seen that once the number of points goes above 500, the solutions become dominated completely. It is also interesting that the search history becomes sparse as well. This figure shows that increasing the number of points extracted from the 3D hand model does not necessarily decrease the error rate. After 500 points, the error becomes greater, which might be similar to over-fitting in Neural Networks. With more points, the optimisation algorithm might gravitate towards the regions with higher density. For the rest of the experiments, we keep the maximum point at 550 to be able to see the history of the search better.

One might say that the error rate is not very low despite the success of MOPSO+EPD. This is because we did not use any initialisation. MOPSO+EPD starts estimating the hand posture every time from a set of random postures. To see how good the results will be in case of an initialisation, we start with a good initial point for the first posture and re-do the experiment. The results are depicted in Fig. 15. This figure evidently shows that the error becomes significantly better, and the same pattern can be observed when increasing the number of points.

Finally, to investigate how much better the MOPSO+EPD with initial poses is compared to MOPSO+EPD without initial poses, Fig. 16 is provided. It can be seen that the Pareto optimal front obtained when there is an initial pose completely dominates the Pareto optimal front without an initial pose. The distribution of solutions obtained by MOPSO+EPD is significantly better than that of MOPSO across both objectives.

Overall, the results show that MOPSO+EPD is able to dominate the MOPSO algorithm on most of the case studies. It was observed that the Pareto optima front obtained by MOPSO+EPD completely dominates that 

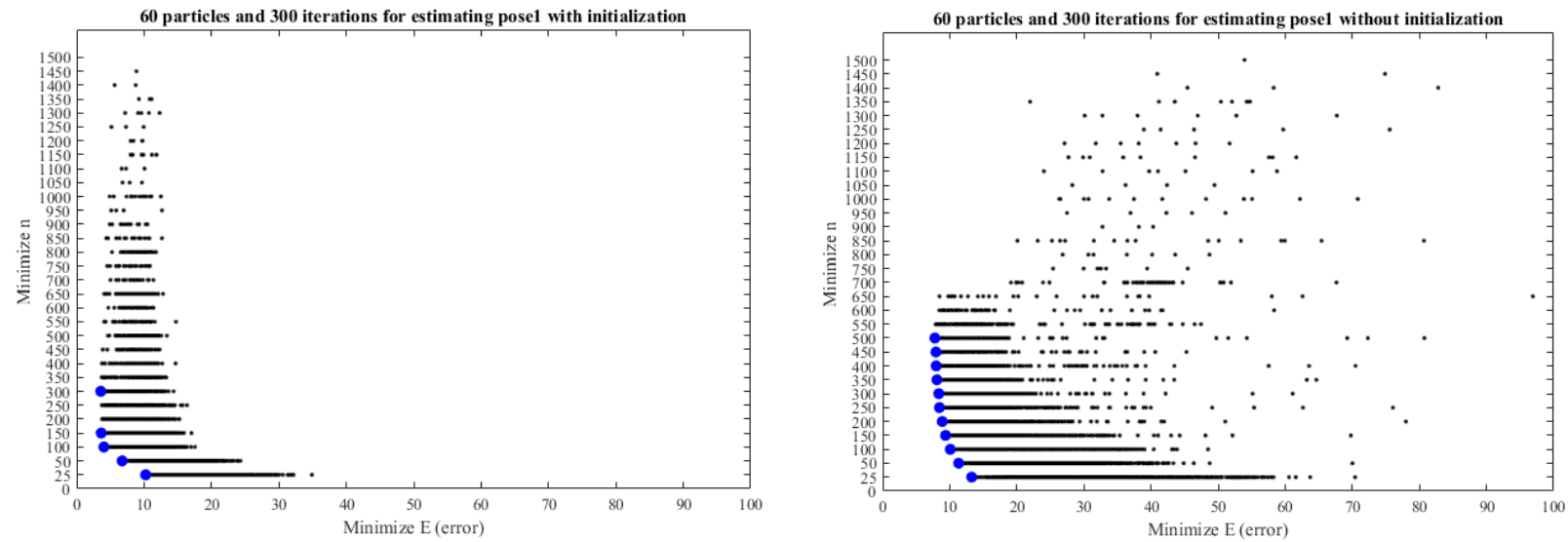

Figure 15: Comparison of MOPSO and MOPSO+EPD on the first posture (left) with and (right) without initialization

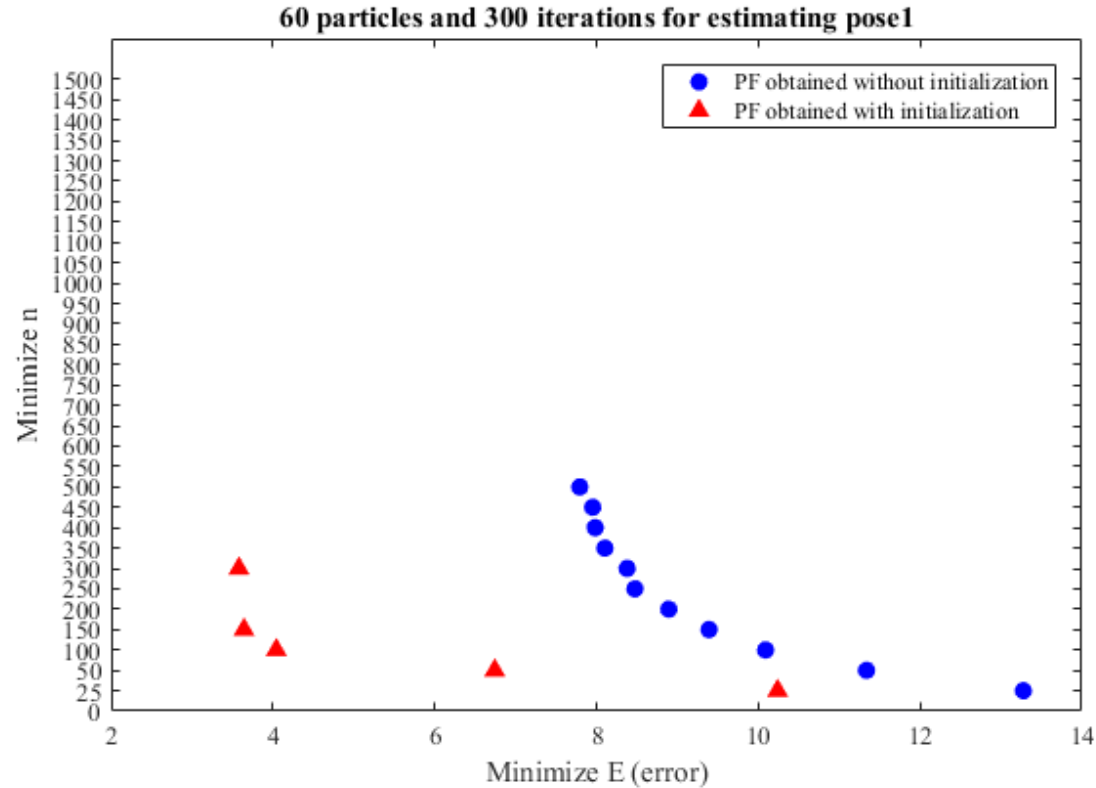

Figure 16: Pareto optimal fronts obtained by MOPSO+EPD (left) with and (right) without initialization 
of MOPSO (e.g. Pose1, Pose8, Pose25, etc.). In the rest of the test cases, a large portion MOPSO's front is dominated by that of MOPSO+EPD. This behaviour can clearly be seen in the Pose8, Pose 35 , etc. case studies which result in having one or two non-dominated solutions with respect to each other. Some of the solutions obtained by MOPSO occasionally dominate one of the solutions found by MOPSO+EPD, but these results show that EPD generally is beneficial for MOPSO for the problem of hand posture estimation.

\subsection{Post analysis of the results}

Due to the high dimensionality of the results (27 variables and 2 objectives), a special tool is required to visualise the results and qualitatively analyse them. Parallel coordinates is used in this subsection [50]. Note that all the parameters and objectives are normalised in $[0,1]$ due to different rages. As the first experiment, the parallel coordinated for the last archive in MOPSO+EPD, which represents the best non-dominated solutions, are visualised in Fig. 17. The main pattern that can be seen is that the first six parameter of all solutions are similar — they are the most influential parameters.

Fig. 17 includes a few solutions, so finding patterns and observing the relationships between parameters and objectives is difficult. In order to better extract qualitative results, the parallel coordinates of all Pareto optimal solutions obtained during the optimisation process of Fig. 15 is shown in Fig. 18. It may be seen in this figure that there are many solutions and we can now analyse the results to find interesting patterns. Since these solutions are the best solutions for this problem, we can 'innovise' (understand) [51] design principals for this problem.

The first interesting pattern is that some of the solutions are fluctuated significantly in some of the parameters: p7, p17 - p27. This means that the solutions are distributed widely on the aforementioned parameters. On the other hand, some of the parameters, particularity P1 - p6 show less distribution. This shows that the impact of the first six variables in calculating the objective functions is more significant. These variables define translation and rotation. Therefore, they have substantial impacts on the objective values compared to the changes in the joint of skeleton. These results show that the applying EPD to p7 to p27 was a good decision since fluctuations in these objectives mostly results in having a useless dominated solution.

It was hypothesized in the preceding section that error $(E)$ and number of points in the point cloud $(n)$ are in conflict. To experimentally investigate this, the parallel coordinates are filtered based on low values for $n$ in Fig.
18. The second sub-figure in this figure highlight the filtered coordinates with a dark colour. Inspecting the behaviour of these lines, it is evident that low values of $n$ correspond to high values for $E$. This shows that there two objectives are in conflict and justify that the use of multi-objective optimisation algorithm to solve this problem.

To further investigate whether the objectives are in conflict or not, Fig. 19 is given. This figure filters the parallel coordinates based on maximum $E$. It may be seen that that high values for the first objective result in low values in the second objective. An interesting pattern is the high density of solution in the first few parameters. This shows that there has been a lot of non-domianted solutions that was dominated eventually when MOPSO+EPD found a good range for the variables of translation and rotation.

The results so far show that the objectives are in conflict and the first six variables are very important. This justifies the use of EPD only on skeleton in MOPSO+EPD. Randomly changing translation and rotation is very likely to give a worse solutions and the results show that the approach of this paper is beneficial.

To see which designs lead to minimal E, Fig.20 is provided in this section. This figure filters the parallel coordinates based on low values for $E$. There are several interesting patterns in this figure. The first pattern can be seen in the distribution of solutions along the second objectives. This shows that in order to find a $3 \mathrm{D}$ hand model with minimum $E$, it is not necessarily required to use the maximum number of points in the point cloud. This is against what might be assumed that increasing the number of points will increase the accuracy of hand posture estimation. The second pattern is the existence of several overlapping lines in the first parameters. These results significantly support that the translation and rotation are very important. This also justifies the use of a good initialiser when estimating hand postures. The high distribution of points for the skeleton variables (p7-p27) also supports the fact that the parameters for the joints locally change the skeleton and can be varied significantly while the objectives do not fluctuate.

To show which designs lead to minimal $E$ and minimal $n$ simultaneously, Fig. 21 filter the coordinates based on minimum $E$ and minimum $n$.

It can be seen that there are a few solutions that show low values for $E$ and require a small number of points in the point cloud. It is interesting that all solutions have the same values for the first six parameters. This is consistent with the observations in the preceding para- 


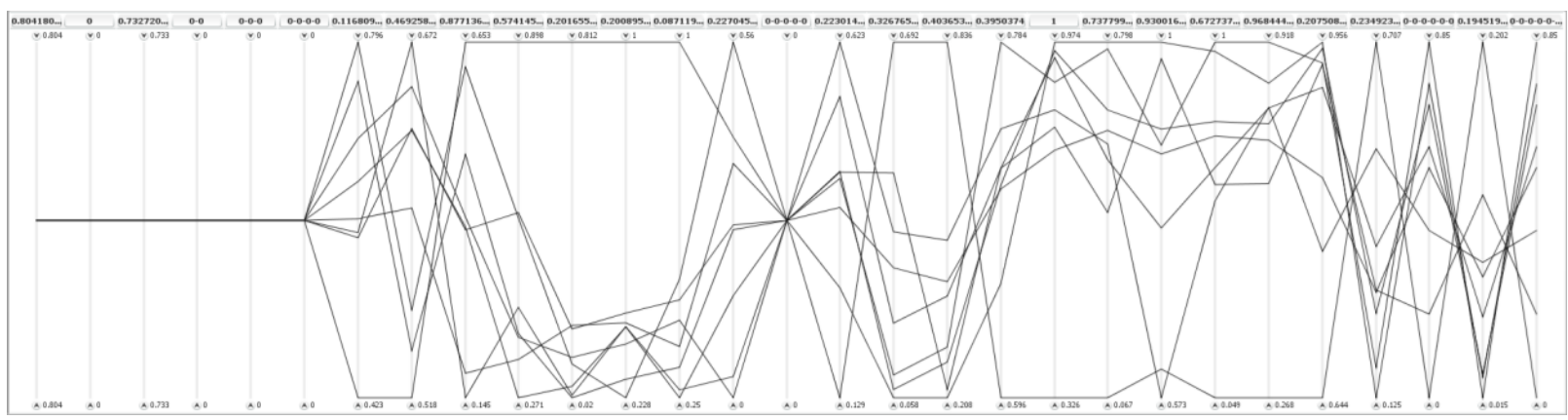

Figure 17: Parallel coordinates for the last archive. There is not much qualitative results can be extract since we have very few solutions.
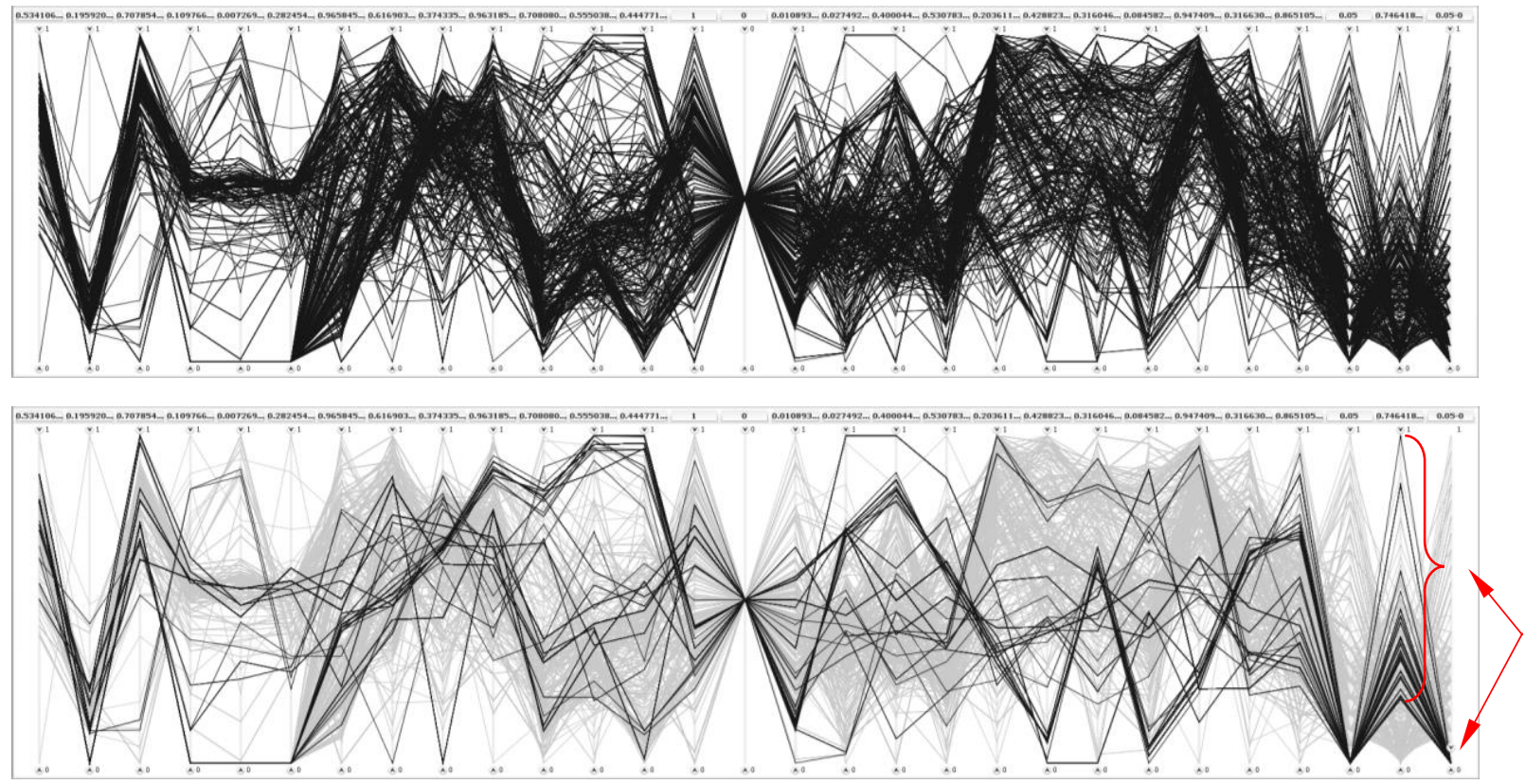

Figure 18: Parallel coordinates of all non-dominated solutions. The first sub-figure shows all the non-dominated solutions. The second sub-figure filters data based on the number of points (last column). Conflicting behaviour is observed since low values for $n$ leads to high value for $E$

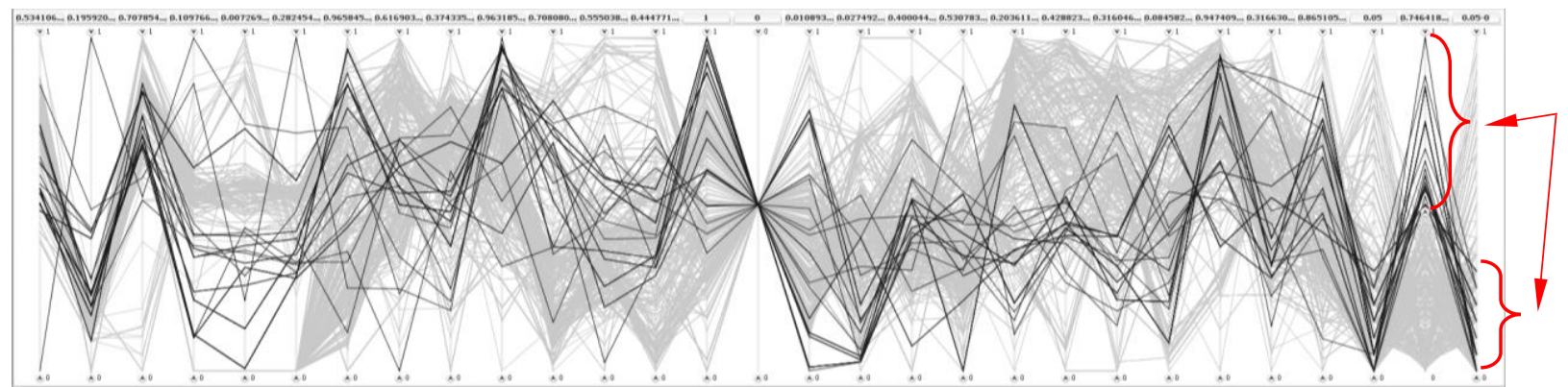

Figure 19: Parallel coordinates when filtering the error $(E)$. Conflicting behaviour of objectives are clearly observed. 


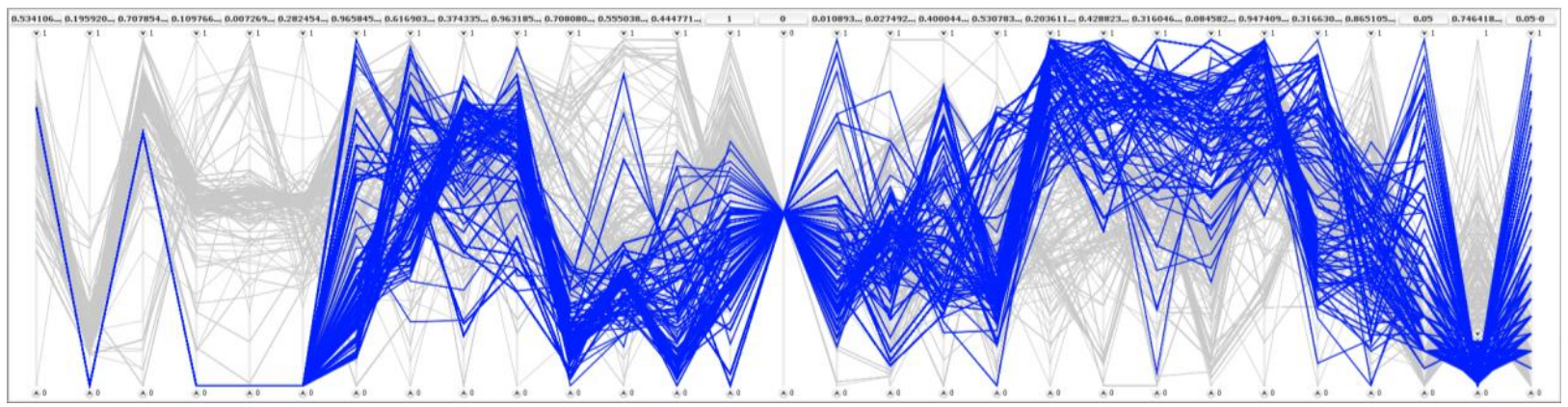

Figure 20: Parallel coordinates when filtering the error $(E)$. The low density of solutions in the first three variables is evident ; showing the radical changes in the objective function when tuning the parameters for translation and rotation.

graphs - the variables that defined the global position and orientation of hand substantially impacts on the objective values.

The last parallel coordinates are illustrated in Fig. 22.

This figure also includes three multi-dimensional scaling using Spring, Sammon, and t-SNE algorithms. Two solutions with the maximum and minimum $E$ are highlighted. As expected, the maximum error is for the solution with the least number of sampled points in the point cloud as can be seen in the yellow line in Fig. 22. However, the solution that shows minimum $E$ does not require maximum number of points in the point cloud, The red line in Fig. 22 shows that the required number of points is reasonably low to get the minimum error. The distribution of solutions between the best and worst solutions (considering $E$ only) in the Spring, Sammon, and t-SNE diagrams are interesting as well. The most interesting fact is that the solution with minimum $E$ is in the centre of figure when using the Spring multidimensional scaling method.

\section{Conclusion}

This work formulated the problem of hand posture estimation as a two-objective problem. An improved version of MOPSO using the concepts of EPD was proposed to solve this problem. The MOPSO algorithm was required to significantly change some of the variables due to the different type of parameters in this problem. To demonstrate the efficiency of the proposed MOPSO+EPD algorithm, ZDT and CEC2009 test suites were employed. The quantitative and qualitative results were compared with MOPSO, NSGA-II, and MOEA/D. The experimental results showed that EPD improves the exploration and coverage of MOPSO significantly. It was also observed that the exploitation and convergence of MOPSO+EPD does not degrade signifi- cantly as compared to MOPSO despite an emphasize on exploratory behaviour.

The second part of the paper was dedicated to solving the problem of hand posture estimation. MOPSO+EPD was employed to estimate the true Pareto optimal front for 50 different postures extracted from five standard datasets. The results showed that MOPSO+EPD finds better Pareto optimal solutions compared to MOPSO for this problem. The post analysis of the results revealed a number of facts (design principals) about the parameters and objectives of this problem.

For future work, it is recommend to use other constraints handling techniques to improve the performance of MOPSO+EPD even further. Devising novel approaches to automatically perform decision making after finding the Pareto optimal solutions set would be a valuable contribution as well.

\section{References}

[1] Matej Črepinšek, Shih-Hsi Liu, and Marjan Mernik. Exploration and exploitation in evolutionary algorithms: A survey. ACM Computing Surveys (CSUR), 45(3):35, 2013.

[2] Swagatam Das, Sayan Maity, Bo-Yang Qu, and Ponnuthurai Nagaratnam Suganthan. Real-parameter evolutionary multimodal optimizationa survey of the state-of-the-art. Swarm and Evolutionary Computation, 1(2):71-88, 2011.

[3] Waqquas A Bukhsh, Andreas Grothey, Ken IM McKinnon, and Paul A Trodden. Local solutions of the optimal power flow problem. IEEE Transactions on Power Systems, 28(4):4780-4788, 2013.

[4] Carlos A Coello Coello. Constraint-handling techniques used with evolutionary algorithms. In Proceedings of the 2016 on Genetic and Evolutionary Computation Conference Companion, pages 563-587. ACM, 2016.

[5] R Timothy Marler and Jasbir S Arora. Survey of multi-objective optimization methods for engineering. Structural and multidisciplinary optimization, 26(6):369-395, 2004.

[6] Seyedali Mirjalili, Andrew Lewis, and Sanaz Mostaghim. Confidence measure: a novel metric for robust meta-heuristic optimisation algorithms. Information Sciences, 317:114-142, 2015. 
[7] Ali Erol, George Bebis, Mircea Nicolescu, Richard D Boyle, and Xander Twombly. Vision-based hand pose estimation: A review. Computer Vision and Image Understanding, 108(1):5273, 2007.

[8] Shanxin Yuan, Guillermo Garcia-Hernando, Bjorn Stenger, Gyeongsik Moon, Ju Yong Chang, Kyoung Mu Lee, Pavlo Molchanov, Jan Kautz, Sina Honari, Liuhao Ge, et al. 3d hand pose estimation: From current achievements to future goals. arXiv preprint arXiv:1712.03917, 2017.

[9] Soniya Lalwani, Sorabh Singhal, Rajesh Kumar, and Nilama Gupta. A comprehensive survey: Applications of multi-objective particle swarm optimization (mopso) algorithm. Transactions on Combinatorics, 2(1):39-101, 2013.

[10] Siddharth S Rautaray and Anupam Agrawal. Vision based hand gesture recognition for human computer interaction: a survey. Artificial Intelligence Review, 43(1):1-54, 2015.

[11] Chengde Wan, Thomas Probst, Luc Van Gool, and Angela Yao. Crossing nets: Dual generative models with a shared latent space for hand pose estimation. arXiv preprint arXiv:1702.03431, 2017.

[12] Martin de La Gorce, David J Fleet, and Nikos Paragios. Model-based 3d hand pose estimation from monocular video. IEEE transactions on pattern analysis and machine intelligence, 33(9):1793-1805, 2011.

[13] Iason Oikonomidis, Nikolaos Kyriazis, and Antonis A Argyros. Efficient model-based 3d tracking of hand articulations using kinect. In $B M V C$, volume 1, page 3, 2011.

[14] Yuhui Shi et al. Particle swarm optimization: developments, applications and resources. In evolutionary computation, 2001. Proceedings of the 2001 Congress on, volume 1, pages 81-86. IEEE, 2001.

[15] Chen Qian, Xiao Sun, Yichen Wei, Xiaoou Tang, and Jian Sun. Realtime and robust hand tracking from depth. In Proceedings of the IEEE Conference on Computer Vision and Pattern Recognition, pages 1106-1113, 2014.

[16] Jay M Wong, Vincent Kee, Tiffany Le, Syler Wagner, GianLuca Mariottini, Abraham Schneider, Lei Hamilton, Rahul Chipalkatty, Mitchell Hebert, David Johnson, et al. Segicp: Integrated deep semantic segmentation and pose estimation. arXiv preprint arXiv:1703.01661, 2017.

[17] Cem Keskin, Furkan Kıraç, Yunus Emre Kara, and Lale Akarun. Real time hand pose estimation using depth sensors. In Consumer Depth Cameras for Computer Vision, pages 119-137. Springer, 2013.

[18] Philip Krejov, Andrew Gilbert, and Richard Bowden. Guided optimisation through classification and regression for hand pose estimation. Computer Vision and Image Understanding, 155:124-138, 2017.

[19] Donghoon Lee, Ming-Hsuan Yang, and Songhwai Oh. Head and body orientation estimation using convolutional random projection forests. IEEE Transactions on Pattern Analysis and Machine Intelligence, 2017.

[20] Pasquale Coscia, Francesco AN Palmieri, Francesco Castaldo, and Alberto Cavallo. 3-d hand pose estimation from kinects point cloud using appearance matching. In Advances in Neural Networks, pages 37-45. Springer, 2016.

[21] Luca Ballan, Aparna Taneja, Jürgen Gall, Luc Van Gool, and Marc Pollefeys. Motion capture of hands in action using discriminative salient points. In European Conference on Computer Vision, pages 640-653. Springer, 2012.

[22] Alex D Holub, Max Welling, and Pietro Perona. Hybrid generative-discriminative visual categorization. International Journal of Computer Vision, 77(1):239-258, 2008

[23] Toby Sharp, Cem Keskin, Duncan Robertson, Jonathan Taylor, Jamie Shotton, David Kim, Christoph Rhemann, Ido Leichter,
Alon Vinnikov, Yichen Wei, et al. Accurate, robust, and flexible real-time hand tracking. In Proceedings of the $33 \mathrm{rd} \mathrm{An-}$ nual ACM Conference on Human Factors in Computing Systems, pages 3633-3642. ACM, 2015.

[24] Philip Krejov, Andrew Gilbert, and Richard Bowden. Combining discriminative and model based approaches for hand pose estimation. In Automatic Face and Gesture Recognition (FG), 2015 11th IEEE International Conference and Workshops on, volume 1, pages 1-7. IEEE, 2015.

[25] Jonathan , Lucas Bordeaux, Thomas Cashman, Bob Corish, Cem Keskin, Toby Sharp, Eduardo Soto, David Sweeney, Julien Valentin, Benjamin Luff, et al. Efficient and precise interactive hand tracking through joint, continuous optimization of pose and correspondences. ACM Transactions on Graphics (TOG), 35(4):143, 2016.

[26] Srinath Sridhar, Franziska Mueller, Antti Oulasvirta, and Christian Theobalt. Fast and robust hand tracking using detectionguided optimization. In Proceedings of the IEEE Conference on Computer Vision and Pattern Recognition, pages 3213-3221, 2015.

[27] Anastasia Tkach, Mark Pauly, and Andrea Tagliasacchi. Spheremeshes for real-time hand modeling and tracking. ACM Transactions on Graphics (TOG), 35(6):222, 2016

[28] Anastasia Tkach, Andrea Tagliasacchi, Edoardo Remelli, Mark Pauly, and Andrew Fitzgibbon. Online generative model personalization for hand tracking. ACM Transactions on Graphics (TOG), 36(6):243, 2017.

[29] CA Coello Coello. Evolutionary multi-objective optimization: a historical view of the field. IEEE computational intelligence magazine, 1(1):28-36, 2006.

[30] Francis Ysidro Edgeworth. Mathematical psychics: An essay on the application of mathematics to the moral sciences, volume 10. Kegan Paul, 1881.

[31] Vilfredo Pareto. Cours d'économie politique, volume 1. Librairie Droz, 1964.

[32] Carlos A Coello Coello. Evolutionary multi-objective optimization: some current research trends and topics that remain to be explored. Frontiers of Computer Science in China, 3(1):18-30, 2009.

[33] C.A.C. Coello, G.T. Pulido, and M.S. Lechuga. Handling multiple objectives with particle swarm optimization. Evolutionary Computation, IEEE Transactions on, 8(3):256-279, 2004.

[34] Margarita Reyes-Sierra and CA Coello Coello. Multi-objective particle swarm optimizers: A survey of the state-of-the-art. International journal of computational intelligence research, 2(3):287-308, 2006.

[35] Marcus Randall and Andrew Lewis. An extended extremal optimisation model for parallel architectures. In e-Science and Grid Computing, 2006. e-Science'06. Second IEEE International Conference on, pages 114-114. IEEE, 2006.

[36] Andrew Lewis, David Abramson, and Tom Peachey. An evolutionary programming algorithm for automatic engineering design. Parallel Processing and Applied Mathematics, pages 586594, 2004.

[37] Per Bak and Kan Chen. Self-organized criticality. Scientific American;(United States), 264(1), 1991.

[38] Andrew Lewis, David Abramson, and Tom Peachey. An evolutionary programming algorithm for automatic engineering design. In International Conference on Parallel Processing and Applied Mathematics, pages 586-594. Springer, 2003.

[39] David E Goldberg and John H Holland. Genetic algorithms and machine learning. Machine learning, 3(2):95-99, 1988.

[40] Per Bak. how nature works. na, 2002.

[41] James Kennedy. Particle swarm optimization. In Encyclopedia of machine learning, pages 760-766. Springer, 2011. 
[42] Eckart Zitzler, Kalyanmoy Deb, and Lothar Thiele. Comparison of multiobjective evolutionary algorithms: Empirical results. Evolutionary computation, 8(2):173-195, 2000.

[43] Qingfu Zhang, Aimin Zhou, Shizheng Zhao, Ponnuthurai Nagaratnam Suganthan, Wudong Liu, and Santosh Tiwari. Multiobjective optimization test instances for the cec 2009 special session and competition. University of Essex, Colchester, UK and Nanyang technological University, Singapore, special session on performance assessment of multi-objective optimization algorithms, technical report, 264, 2008.

[44] Kalyanmoy Deb, Amrit Pratap, Sameer Agarwal, and TAMT Meyarivan. A fast and elitist multiobjective genetic algorithm: Nsga-ii. IEEE transactions on evolutionary computation, 6(2):182-197, 2002.

[45] Qingfu Zhang and Hui Li. Moea/d: A multiobjective evolutionary algorithm based on decomposition. IEEE Transactions on evolutionary computation, 11(6):712-731, 2007.

[46] Margarita Reyes Sierra and Carlos A Coello Coello. Improv ing pso-based multi-objective optimization using crowding, mutation and-dominance. In International Conference on Evolutionary Multi-Criterion Optimization, pages 505-519. Springer, 2005.

[47] Jason R Schott. Fault tolerant design using single and multicriteria genetic algorithm optimization. Technical report, DTIC Document, 1995.

[48] Chi Keong Goh and Kay Chen Tan. An investigation on noisy environments in evolutionary multiobjective optimization. IEEE Transactions on Evolutionary Computation, 11(3):354381, 2007.

[49] Gregorio Toscano Pulido and Carlos A Coello Coello. A constraint-handling mechanism for particle swarm optimization. In Evolutionary Computation, 2004. CEC2004. Congress on, volume 2, pages 1396-1403. Ieee, 2004.

[50] Wang Hu and Gary G Yen. Adaptive multiobjective particle swarm optimization based on parallel cell coordinate system. IEEE Transactions on Evolutionary Computation, 19(1):1-18, 2015.

[51] Kalyanmoy Deb and Aravind Srinivasan. Innovization: Innovating design principles through optimization. In Proceedings of the 8th annual conference on Genetic and evolutionary computation, pages 1629-1636. ACM, 2006.

[52] Jonathan Tompson, Murphy Stein, Yann Lecun, and Ken Perlin. Real-time continuous pose recovery of human hands using convolutional networks. ACM Transactions on Graphics, 33, August 2014

[53] Srinath Sridhar, Antti Oulasvirta, and Christian Theobalt. Interactive markerless articulated hand motion tracking using rgb and depth data. In Proceedings of the IEEE International Conference on Computer Vision (ICCV), December 2013.

\section{Appendix}

Dataset used:

Complete results of MOPSO+EPD on the case studies: 


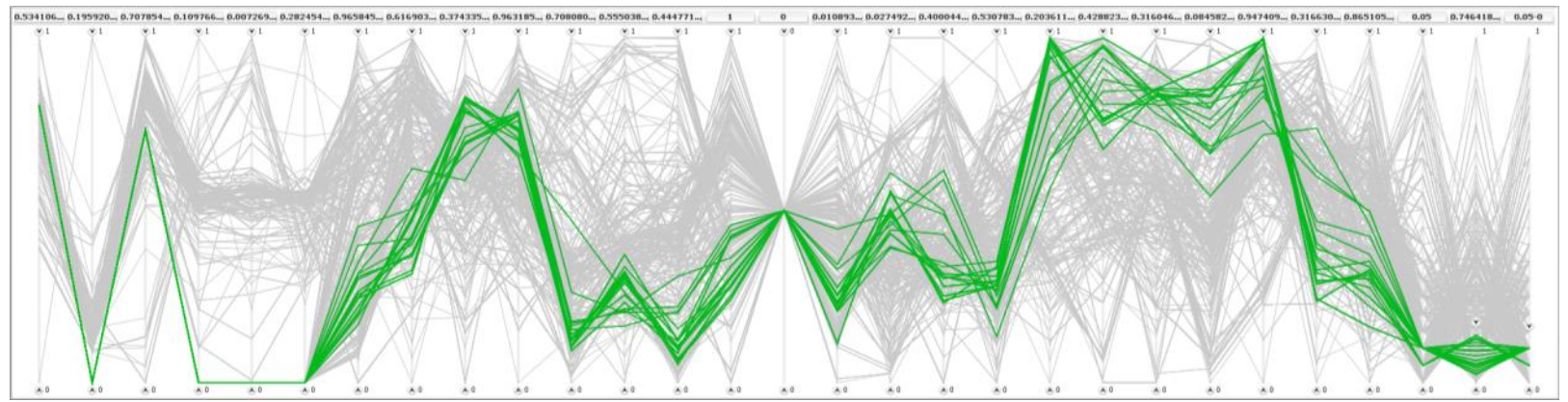

Figure 21: Parallel coordinates when filtering both objectives. Low density of solutions in the first six variables is observed again. Fluctuation of the results in the rest of parameters is interesting as well.

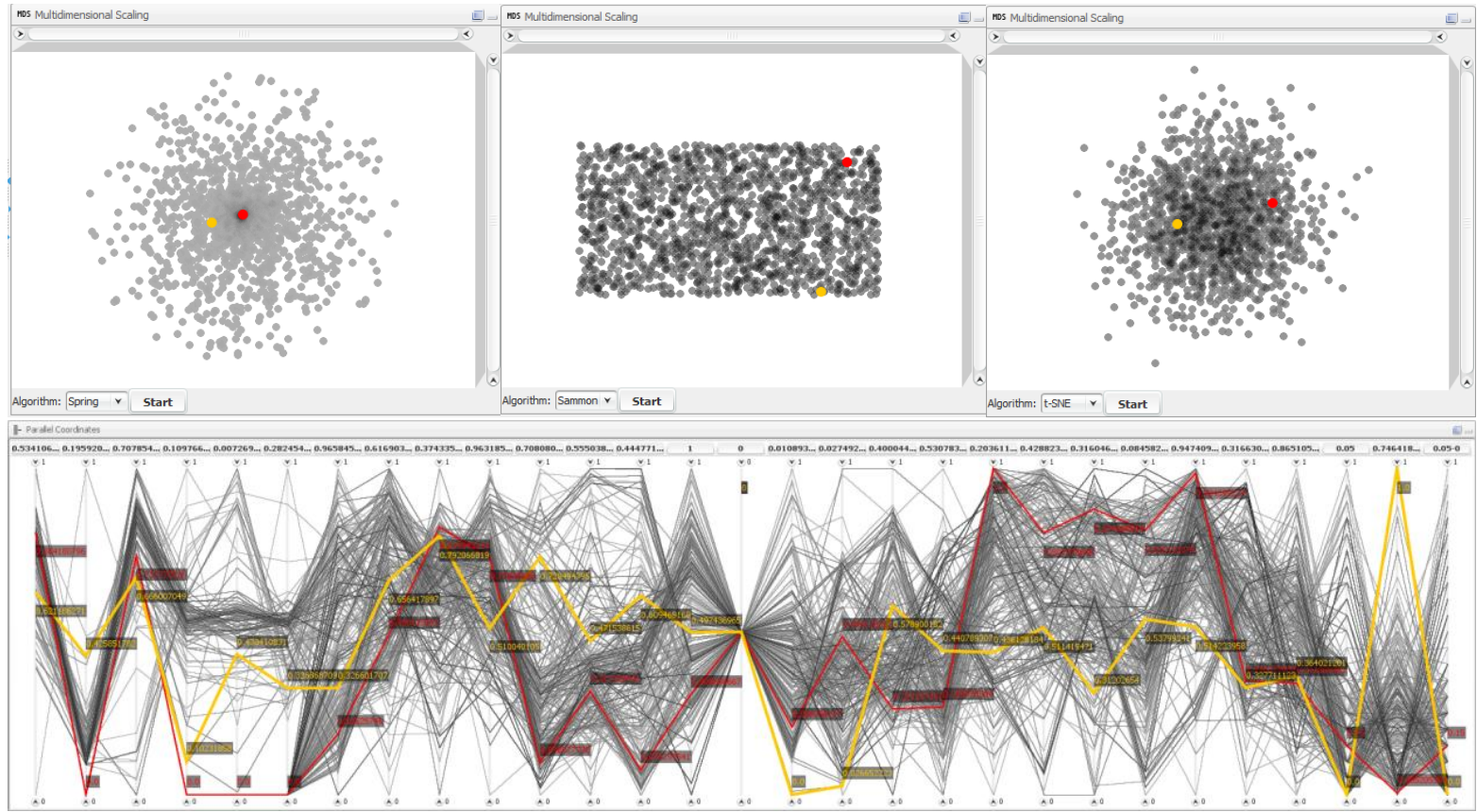

Figure 22: Parallel coordinates and multi-dimensional scaling using Spring, Sammon, and t-SNE algorithms from left to right. This figure highlights the solution with maximum $\mathrm{E}$ and the solution with minimum $\mathrm{E}$. The distribution of the solutions between these two points are interesting. 


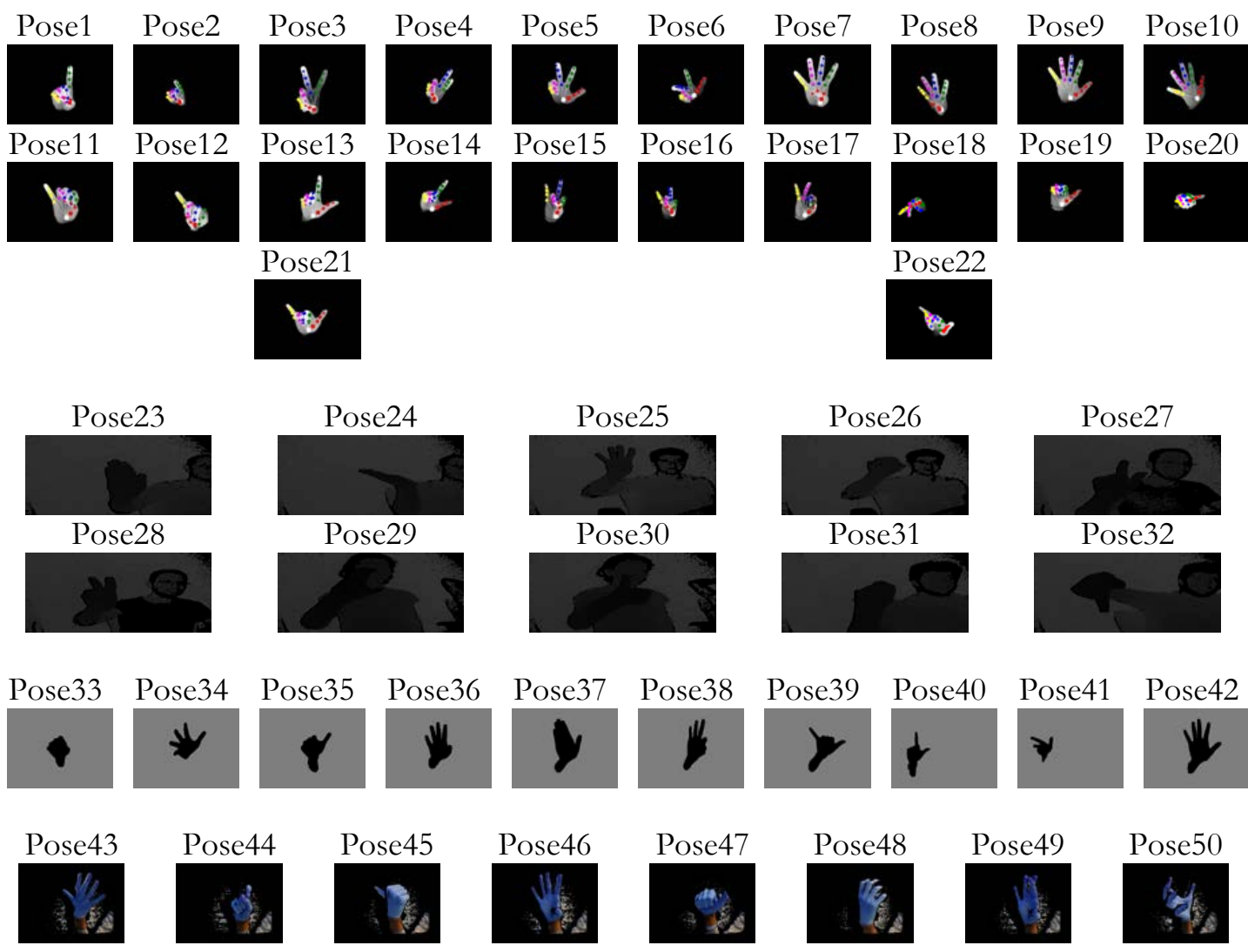

Figure 23: Case studies: 50 postures extracted from five datasets [52, 53, 27] 

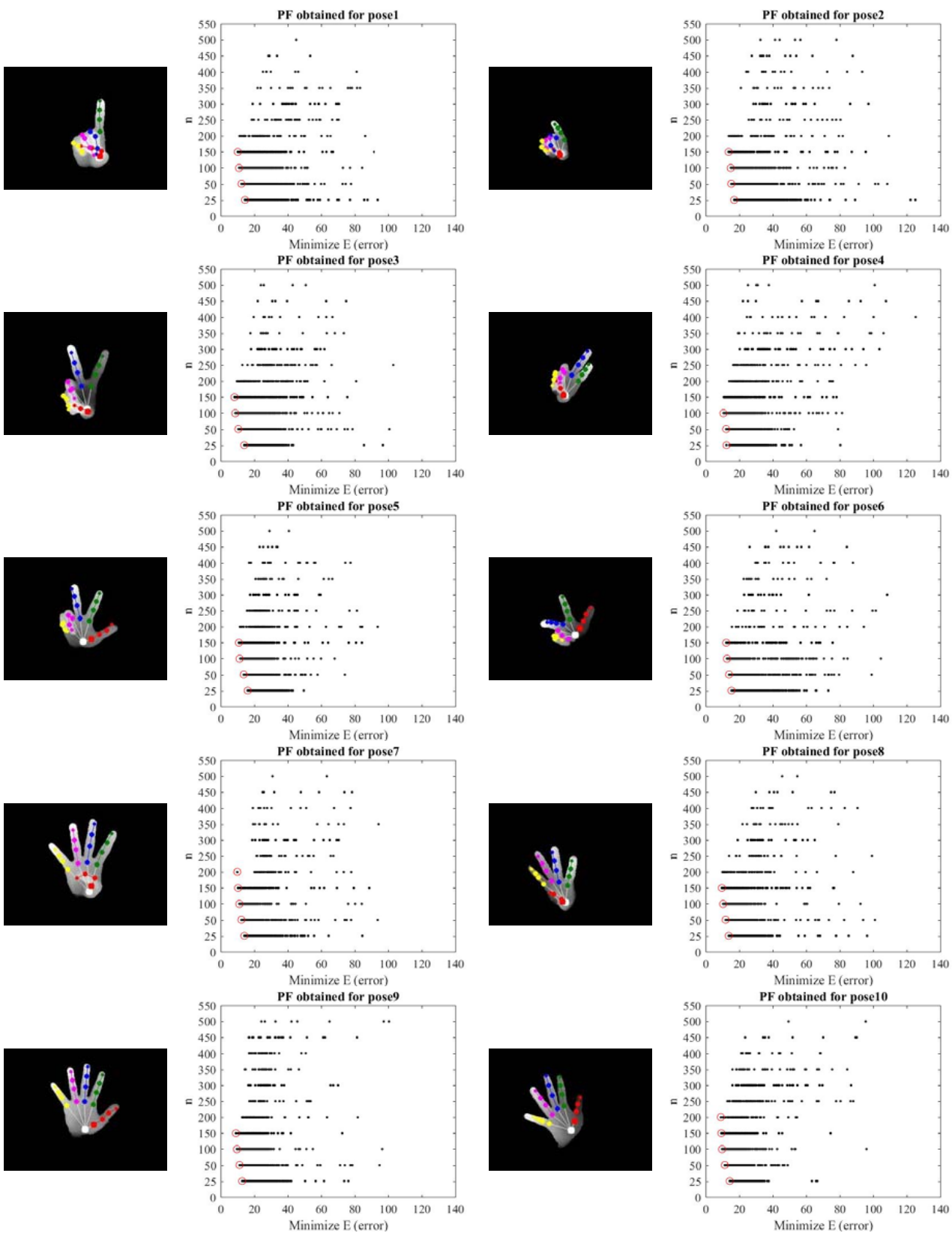

Figure 24: Poses extracted from the dataset 1 

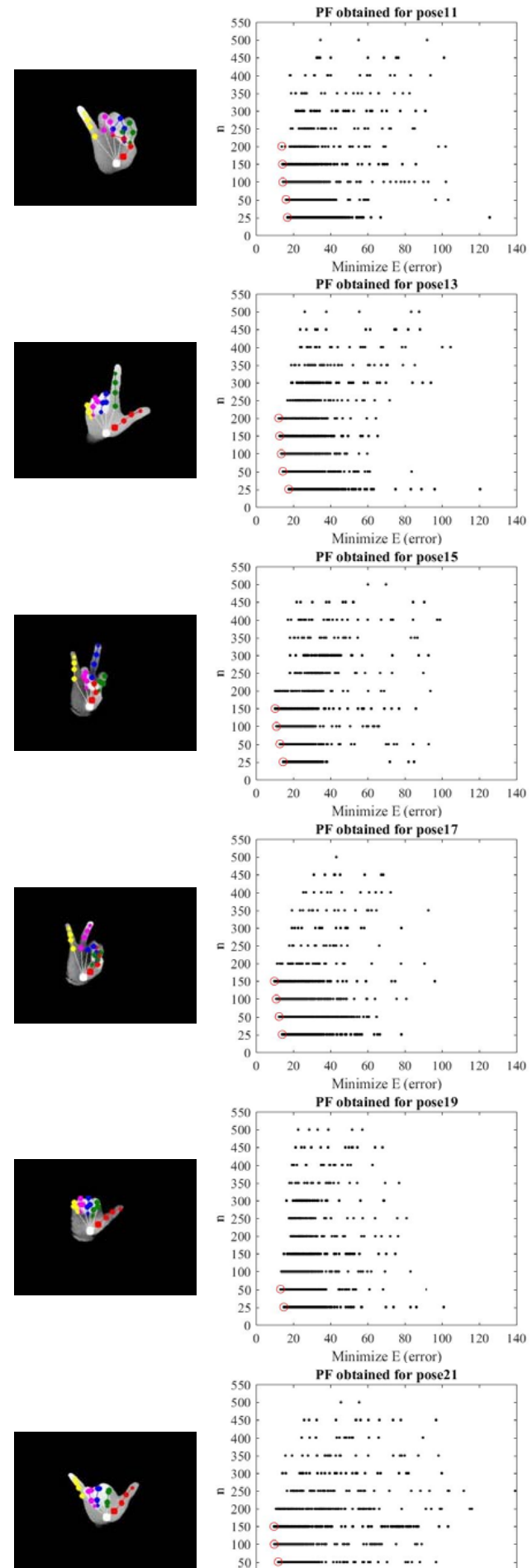
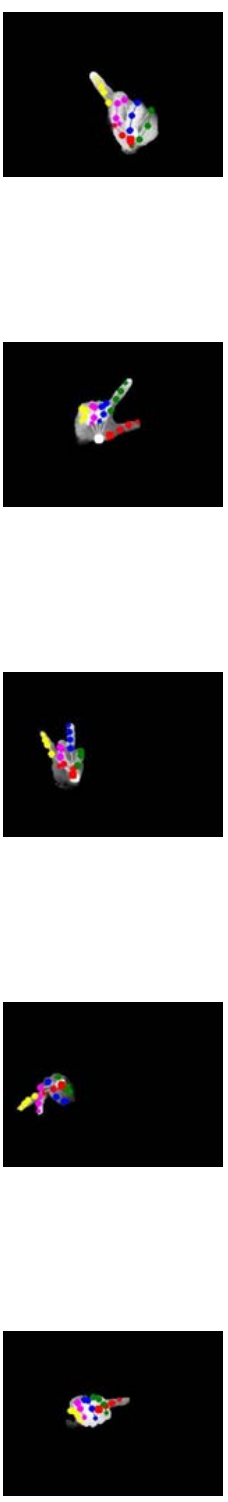

PF obtained for pose12
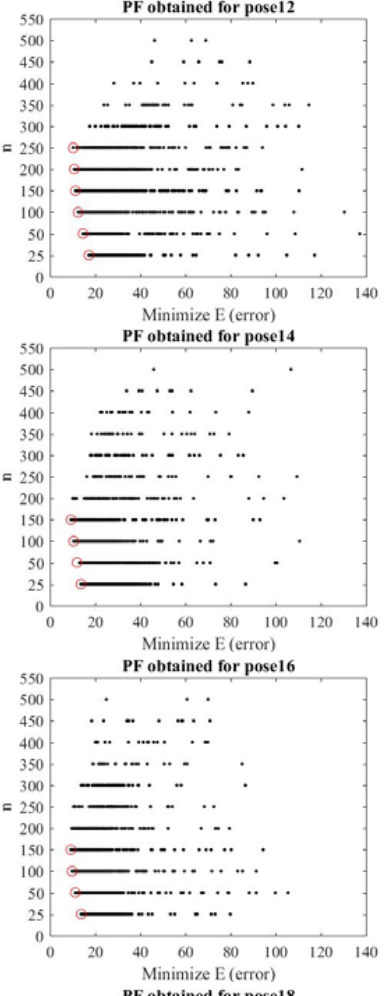

PF obtained for pose18

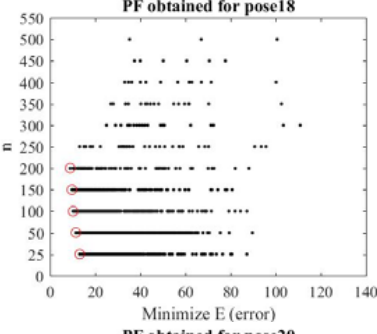
PF obtained for pose20

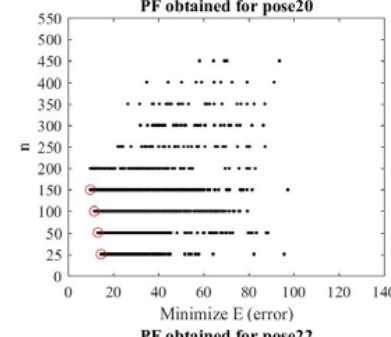
PF obtained for pose22
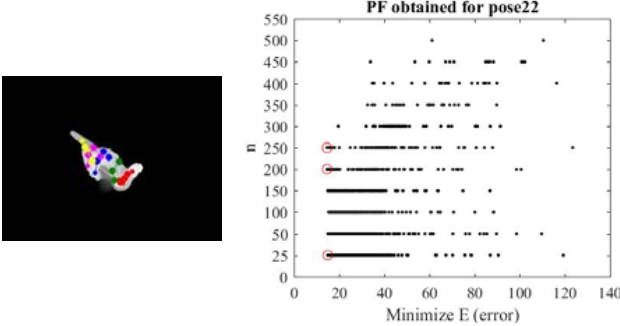

Figure 25: Poses extracted from the dataset 1 


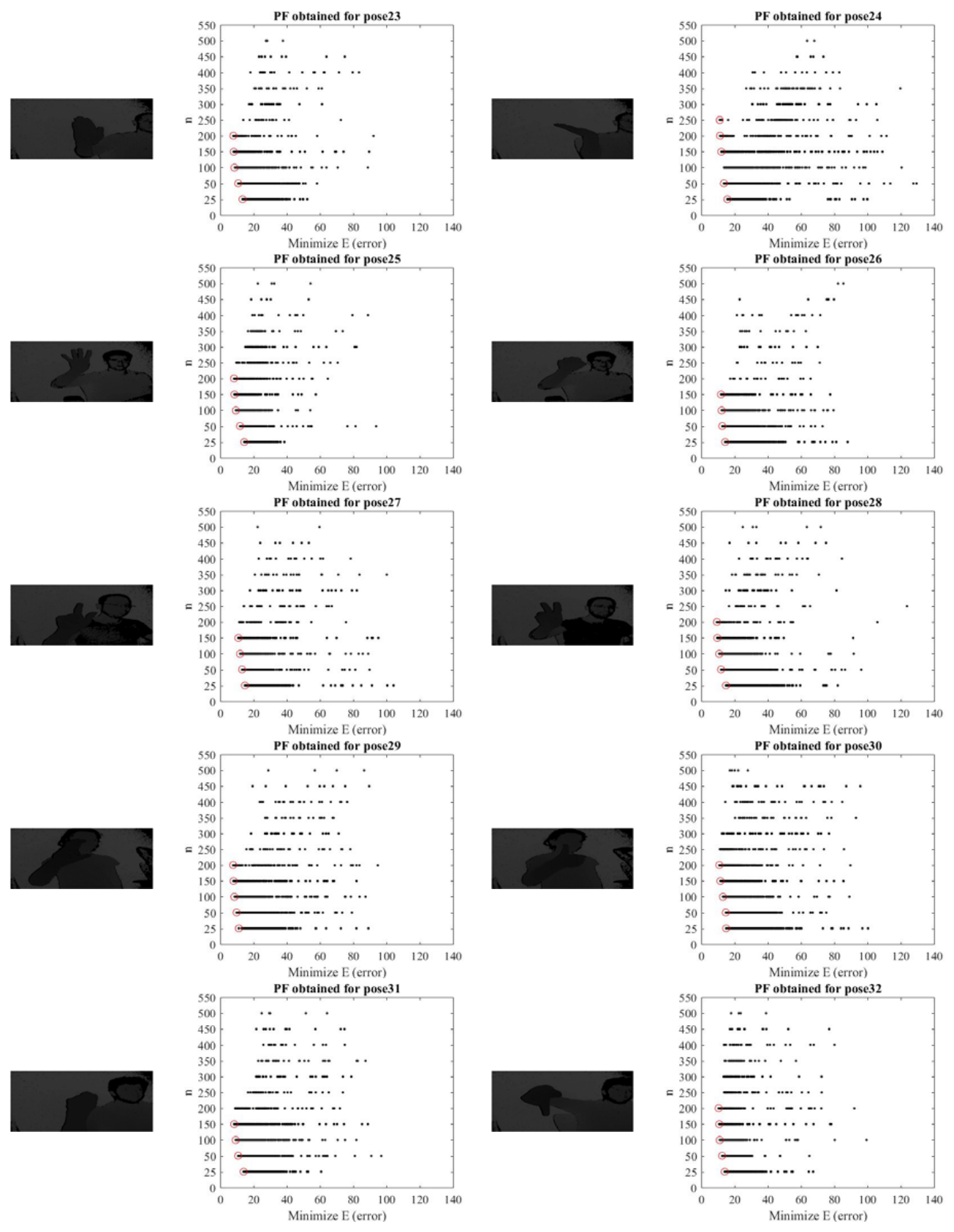

Figure 26: Poses extracted from the dataset 1 


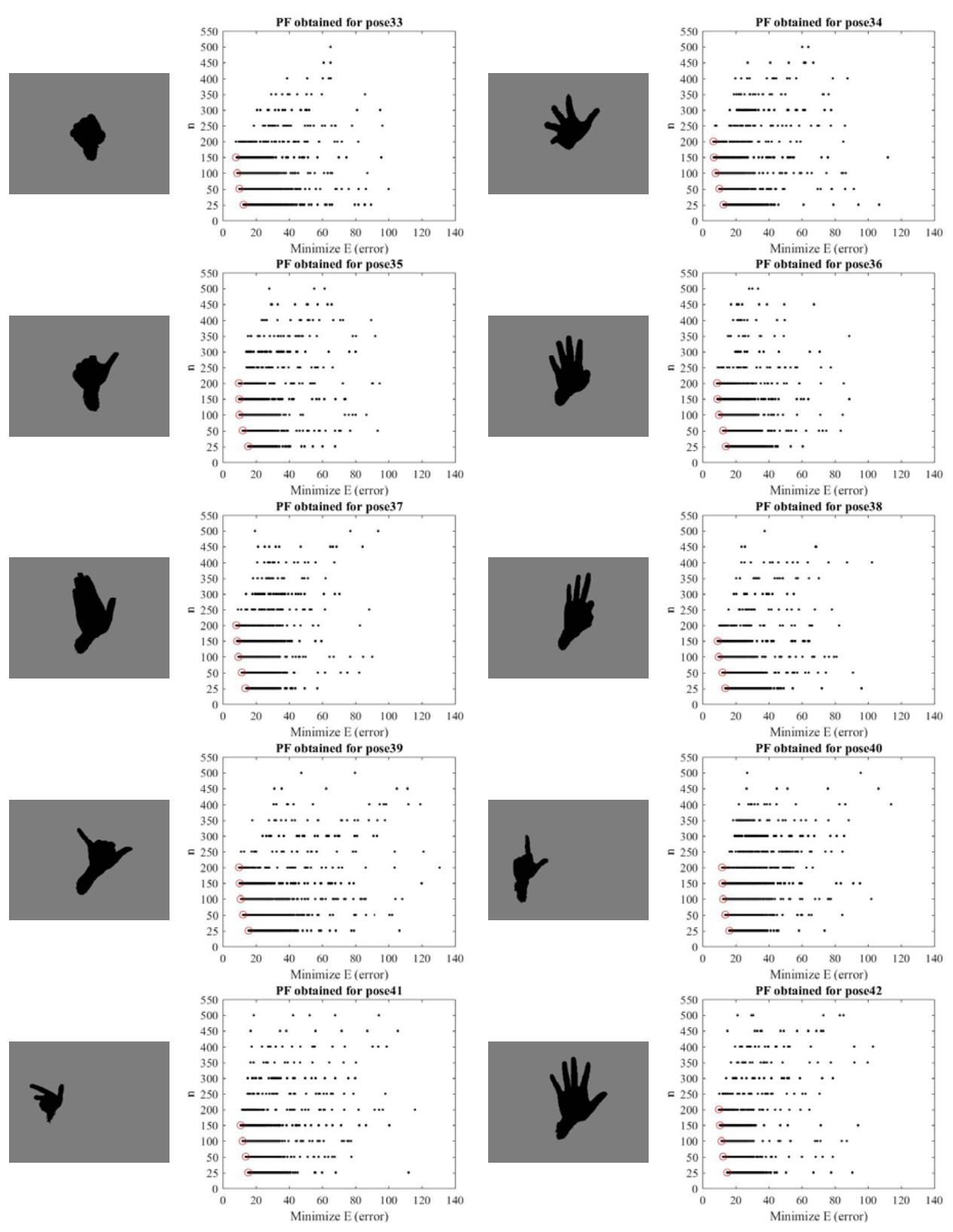

Figure 27: Poses extracted from the dataset 1 

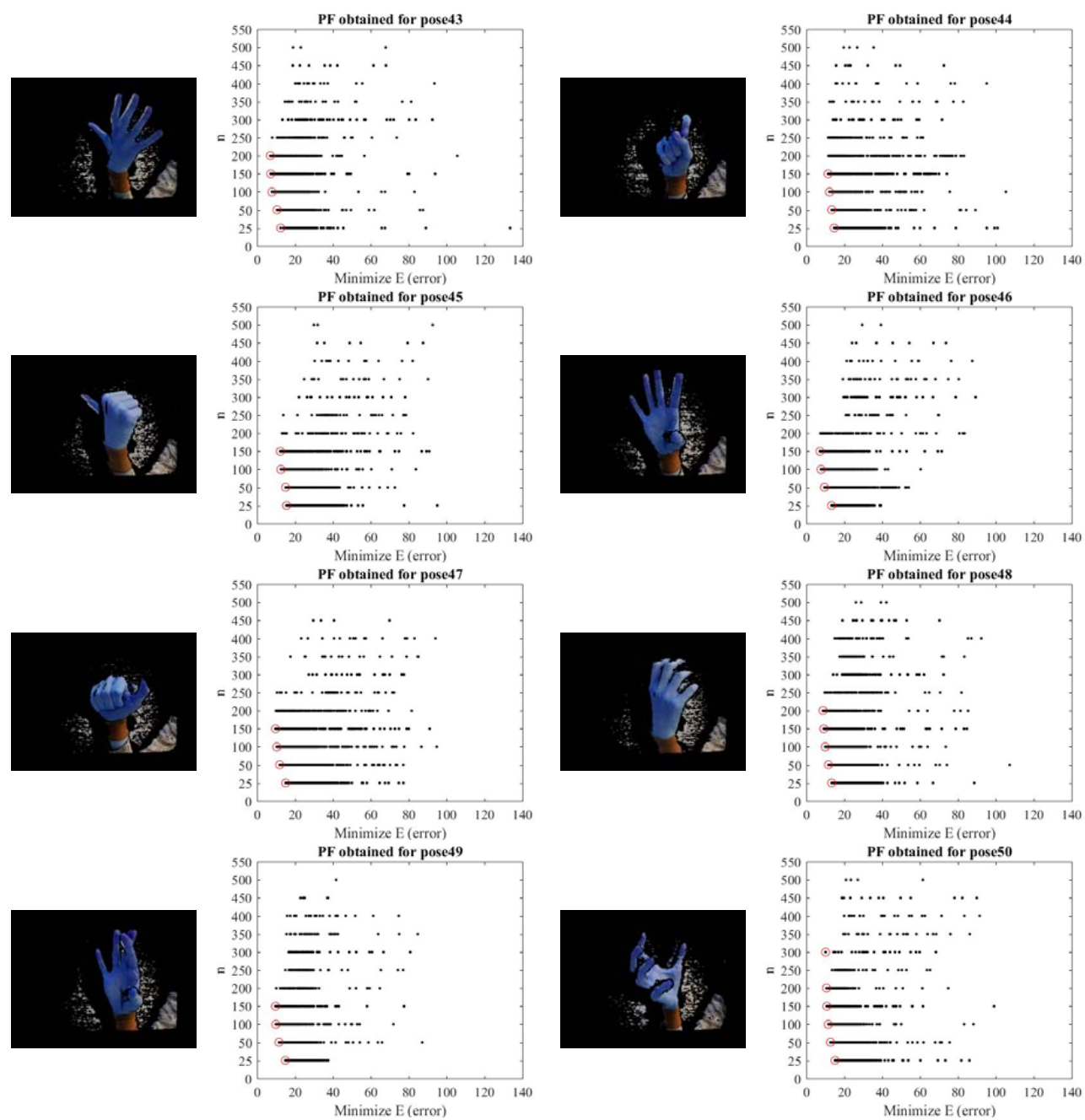

Figure 28: Poses extracted from the dataset 1 